\title{
High-order X-FEM for the simulation of sound absorbing poro-elastic materials with coupling interfaces
}

\author{
Shaoqi $\mathrm{Wu}^{a}$, Olivier Dazel ${ }^{b}$, Gwénaël Gabard ${ }^{b}$, Grégory Legrain $^{a}$ \\ ${ }^{a}$ Institut de Recherche en Génie Civil et Mécanique (UMR CNRS 6183), École Centrale de Nantes, Université de \\ Nantes, 44321 Nantes, France \\ ${ }^{b}$ Laboratoire d'Acoustique de l'Université du Mans (UMR CNRS 6613), Le Mans Université, 72085 Le Mans \\ Cedex, France
}

\begin{abstract}
SUMMARY
In this paper, the acoustic field with the presence of poro-elastic materials is simulated by the eXtended Finite Element Method (X-FEM). Problems involving interfaces between different media are our main focus. The proposed method allows interfaces to be embedded in the finite elements, easing significantly the discretization, especially when the geometry of the interface is complex. The gradient discontinuity at the interface is handled through the ridge enrichment function. The strategies of spatial discretization for two different types of coupling interface are provided. A high-order approximation is used to improve the rate of convergence for the Biot mixed formulation $(\mathbf{u}, p)$ and to eliminate the pollution effect at high frequencies. The verification of the method is performed with two benchmarks. Convergences of the solutions exhibit the capability and the accuracy of the present method under different conditions: coupling types, geometric complexity and a wide range of frequency. The applicability and advantage of the method in practical situations are demonstrated by a car cavity problem where part of the geometry is modified without re-meshing. This paper demonstrates that high-order X-FEM is an efficient computational approach for analysing sound-absorbing poro-elastic materials involving complex geometries.
\end{abstract}

KEY WORDS: X-FEM, High order, Biot theory, mixed coupling interfaces

\section{Introduction}

This paper proposes a computational approach based on the eXtended Finite Element Method (XFEM) to predict the acoustic behaviour of porous structures coupled to other media. Poro-elastic materials (PEMs) are widely used to build sound absorbers for practical noise control problems such as panels for room acoustics and sound proofing in aircraft, cars and trains. The absorption of PEMs originates from energy dissipation in the material by means of structural, thermal and viscous effects due to the internal interaction between an elastic solid phase and the pores of a fluid phase. To describe these dissipation effects, two categories of models were developed and investigated, which are the equivalent fluids [1, 2] and coupled formulations based on Biot's theory [3, 4, 5, 6]. The equivalent fluid model supposes that the solid phase is motionless. It corresponds to the case where the frame stiffness is very high and its vibration can be negligible compared to fluid motion under certain high-frequency excitation. In contrast, formulations based on the Biot equations provide a complete description of the propagation of coupled waves in the elastic and fluid phases within the materials. This is valid the most general model and it is used in the present 15 paper. 
Numerical methods for solving the Biot equations can be divided into two principal categories. The first one corresponds to the classical finite-element methods (FEM) based on low-order piecewise polynomials bases and the second one is classified as generalized wave-based methods using exact wave solution as basis functions. The classical FEM suffers from convergence problems with respect to the number of degrees of freedom. This issue stems partly from the build-up of dispersion error (pollution effects [7]) in wave equations, especially at high frequencies and partly from the disparity of scales between the different types of waves appearing in porous materials (see [8]). High-order polynomials elements have been demonstrated to be an efficient way to address these issues in [9, 10]. Wave-based methods have also been developped to solve problems with highly oscillatory solutions. Ultra weak formulation [11, Generalized finite element method (GFEM) 12 or partition of unity finite element method (PUFEM) [13, wave-based method [14 and Discontinuous Galerkin methods [15] were proposed to solve the Helmholtz equation or the Biot equations. Compared to the classical FEM, wave-based methods show a better ability to capture the oscillation of waves with less computational cost. However, more efforts and physical knowledge for the implementation are required since local, exact solutions of the governing equations have to be obtained beforehand, particularly for complicated formulations such the Biot equations studied in [16] and [14. Contrary to wave-based methods, the choice of basis and computation procedure for the high-order FEM is independent of the physical model.

When modelling interfaces between different materials with the classical FEM, the mesh has to coincide with the geometry of interface. A significant effort is needed for this purpose, especially when the geometry of the interface is complex. In the case where the mesh is not conforming with the interface, Lagrangian multipliers [17, penalty methods [18] or Nitsche methods [19] are frequently employed to weakly enforce the continuity across the interface. The eXtended Finite Element Method is an enriched version of the classical FEM that can tackle such limitations of the mesh and the geometry. X-FEM was first proposed to solve crack growth problems without re-meshing [20] and was also applied to deal with material interfaces [21, 22]. The X-FEM with high-order approximations has been studied in [23, 24] and 25] to alleviate the geometrical error. The most relevant prior work to the present paper is probably the use of X-FEM in fluid-structure interaction analysis such as [26] and [27]. In [27, X-FEM is used to track an interface without remeshing and enforce the coupling condition between fluid and structure with Lagrangian multipliers and a mortar method. In the present work, the location of the interface and the coupling conditions are handled simultaneously by the enrichment strategy without extra operators.

The main contribution of this work is to extend the application of X-FEM approach to the acoustic analysis of porous structures with interfaces. The proposed computational method is able to handle multiple medium interfaces and to ensure convergence of the Biot mixed formulation $(\mathbf{u}, p)$ [5] simultaneously. This paper is organised as follows: in the next section, the physical problem is stated describing the governing equations for different media, and two common coupling interface conditions are introduced. In section 3, the idea of the X-FEM and level-set technique are introduced with the construction of a suitable enrichment function. Discretization strategies for two coupling configurations are then detailed. The verification and application of the method are demonstrated by three numerical examples with gradually more complex geometries, considering different materials and frequencies in the last section.

\section{Formulation}

\subsection{Problem statement and variational formulation}

The problem studied in this paper concerns a bounded domain $\Omega$ composed of several acoustic media which have different physical models and material properties. For the purpose of clarity, here a two-media configuration is considered to formulate the equations and interface conditions. 
This can be easily extended to more complex problems with multiple interfaces. In this paper, the problem is studied in the frequency space, focusing on acoustic behaviour of the media in steady state. A harmonic convention $e^{+j \omega t}$ is assumed with angular frequency $\omega$.

The boundary of the domain is denoted $\Gamma$. The domain is divided by a material interface $\Gamma^{*}$ into two subdomains $\Omega_{1}$ and $\Omega_{2}$ as illustrated in Fig. 1. $\mathbf{n}_{1}$ and $\mathbf{n}_{2}$ are the unit outward normal at the interface of each subdomain with $\mathbf{n}_{1}=-\mathbf{n}_{2}$. In each subdomain we can use a different model for the porous material: an acoustic fluid or a poro-elastic material. As a consequence, two common types of interfaces will be discussed in this paper: an interface between an acoustic fluid and a poro-elastic region, and an interface between two different poro-elastic materials. These coupling interfaces are representative of many situations found in practical applications. It should be noted that the acoustic fluid considered in this paper comprises all types of fluid medium which are governed by the classical Helmholtz equation. The equivalent fluid model[1, 2] is therefore included in this class of material.

In order to apply the X-FEM approach to this problem, the variational formulations of the governing equations for each media have to be established.

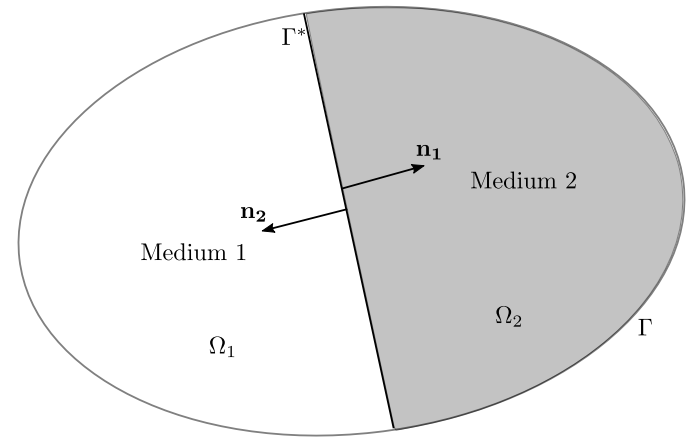

Figure 1: Computational domain with two different media.

\subsubsection{Poro-elastic medium}

The model for the poro-elastic medium used here is the mixed formulation of the Biot equation formulated with pressure $p$ in the fluid phase and the displacement $\mathbf{u}^{s}$ of the solid phase, first proposed in [5. The variational formulation from [28] with test function $q$ for pressure and $\mathbf{v}^{s}$ for the displacement is written as follows:

$$
\begin{gathered}
\int_{\Omega} \hat{\sigma}_{i j}^{s}\left(\mathbf{v}^{s}\right) \varepsilon_{i j}^{s}\left(\mathbf{u}^{s}\right) \mathrm{d} \Omega-\omega^{2} \int_{\Omega} \tilde{\rho} \mathbf{v}_{i}^{s} \mathbf{u}_{i}^{s} \mathrm{~d} \Omega-\int_{\Omega} \tilde{\gamma} \mathbf{v}_{i}^{s} \frac{\partial p}{x_{i}} \mathrm{~d} \Omega-\int_{\Gamma} \mathbf{v}_{i}^{s} \boldsymbol{n}_{j} \hat{\sigma}_{i j}^{s}\left(\mathbf{u}^{s}\right) \mathrm{d} S=0, \\
\int_{\Omega}\left[\frac{1}{\omega^{2} \tilde{\rho}_{e q}} \frac{\partial q}{\partial x_{i}} \frac{\partial p}{\partial x_{i}}-\frac{1}{\tilde{K}_{e q}} q p\right] \mathrm{d} \Omega-\int_{\Omega} \tilde{\gamma} \frac{\partial q}{\partial x_{i}} \mathbf{u}_{i}^{s} \mathrm{~d} \Omega+\int_{\Gamma} q u_{n}^{t} \mathrm{~d} S=0,
\end{gathered}
$$

where $\hat{\sigma}^{s}$ represents the in-vacuo stress tensor of the solid phase and $\varepsilon^{s}$ is the associated strain tensor. The surface term $u_{n}^{t}$ defined on the boundary $\Gamma$ is the normal total displacement of the porous material, which consists of the displacement of elastic solid phase and fluid phase. The coefficients $\tilde{\rho}, \tilde{\rho}_{e q}, \tilde{K}_{e q}$ and $\tilde{\gamma}$ are the effective density of the solid phase, the effective density and compressibility of the fluid phase and coupling parameter, respectively. These parameters originate from the Johnson-Champoux model summarised in Appendix A. Note that the coefficients used in this formulation are slightly different from those used in [5. This formulation is used because the number of degrees of freedoms (dofs in the following) per node is reduced to three instead of four compared to other displacement-based formulations [6] in two-dimensional problems, one for pressure and two for the solid displacement. In addition, this formulation leads to a more natural coupling with acoustic fluids and other PEMs, as explained below. 
Without the terms involving $\tilde{\gamma}$, Eq. (1a) can be considered as the dynamic behaviour of the solid phase in vacuum and Eq. $1 \mathrm{~b}$ is the Helmholtz equation for the equivalent fluid.

\subsubsection{Acoustic fluid medium}

The variational formulation for the acoustic fluid medium is that for the Helmholtz equation with test function $q$ for the pressure:

$$
\int_{\Omega}\left(\frac{1}{\omega^{2} \rho} \frac{\partial q}{\partial x_{i}} \frac{\partial p}{\partial x_{i}}-\frac{1}{K} q p\right) \mathrm{d} \Omega+\int_{\Gamma} q \frac{1}{\omega^{2} \rho} \frac{\partial p}{\partial n} \mathrm{~d} S=0
$$

where $\rho$ is the fluid density and $K$ is its compressibility modulus. The surface integral term on $\Gamma$ corresponds to the normal total displacement of the acoustic fluid. While the Biot equations for the PEM support three distinct waves (two compression waves and a shear wave), only one compression wave propagates in the acoustic fluid.

\subsection{Interface coupling}

Two coupling configurations are now discussed. First we consider the interface conditions between an acoustic fluid (denoted by the subscript 1) and a poro-elastic region (denoted by the subscript 2 ). The conditions to be imposed on such an interface are

$$
\begin{gathered}
\frac{1}{\omega^{2} \rho_{1}} \frac{\partial p_{1}}{\partial \mathbf{n}_{1}}=-\mathbf{u}_{2}^{t} \cdot \mathbf{n}_{2}, \\
p_{1}=p_{2}, \\
\hat{\sigma}_{2}^{s} \cdot \mathbf{n}_{2}=0 .
\end{gathered}
$$

Eq.(3a) represents the continuity of the total normal displacement $\mathbf{u}^{t}$ which is defined as the volume average of the displacement including both fluid and solid phases. Eq. (3b) represents the continuity of pressure. Eq.(3c) corresponds to the free-surface condition on the porous material.

The second type of coupling conditions corresponds to an interface between two different PEMs (denoted by the subscripts 1 and 2):

$$
\begin{gathered}
\mathbf{u}_{1}^{s} \cdot \mathbf{n}_{1}=-\mathbf{u}_{2}^{s} \cdot \mathbf{n}_{2}, \\
\mathbf{u}_{1}^{t} \cdot \mathbf{n}_{1}=-\mathbf{u}_{2}^{t} \cdot \mathbf{n}_{2}, \\
p_{1}=p_{2}, \\
\hat{\sigma}_{1}^{s} \cdot \mathbf{n}_{1}=-\hat{\sigma}_{2}^{s} \cdot \mathbf{n}_{2} .
\end{gathered}
$$

Eq. (4a) to Eq.4d represents, respectively, the continuity of the normal displacement of the solid phase, the continuity of the total normal displacement, the continuity of pressure in the fluid phase, and the continuity of the in-vacuo normal stress in the solid phase.

When applying the above sets of coupling conditions to the variational formulations Eq.(1) and Eq. 22), the surface terms on the interface $\Gamma^{*}$ vanish. In addition, since we use a continuous discretisation of the pressure field, its continuity across the interface is directly enforced. These types of interface conditions are called natural couplings, resulting in a simple implementation since

\section{Spatial discretization with X-FEM}

\subsection{The eXtended Finite Element Method (X-FEM)}

As is customary with the finite element method, the computational domain $\Omega$ is divided into nonoverlapping elements $\Omega_{e}$, constituting a mesh. The general vector field $\mathbf{u}(\mathbf{x})$ is obtained numerically 
by a polynomial approximation on each element $\Omega_{e}$ :

$$
\left.\mathbf{u}(\mathbf{x})\right|_{\Omega_{e}}=\sum_{i=1}^{n} \mathbf{N}_{i}(\mathbf{x}) u_{i}
$$

where $n$ is the number of the degree of freedom on the element, $u_{i}$ is the $i^{\text {th }}$ nodal contribution of this approximation, $\mathbf{N}_{i}(\mathbf{x})$ represents the $i^{\text {th }}$ shape function associated with $u_{i}$, which usually is a piecewise polynomial basis.

When there is an interface between two materials, the classical finite-element method requires a mesh that conforms to the interface in order to obtain the optimal rate of the convergence [22]. In contrast, with the eXtended Finite Element Method (X-FEM) the mesh does not need to coincide with the interface, different materials can overlap within one element. The presence of a material discontinuity is naturally handled by adding an enrichment term to the classical finite element approximation Eq. (5). For every element that intersects with the interface, the displacement field $\mathbf{u}(\mathbf{x})$ is enriched locally by writing $[29]$

$$
\left.\mathbf{u}(\mathbf{x})\right|_{\Omega_{e}}=\sum_{i=1}^{n} \mathbf{N}_{i}(\mathbf{x}) u_{i}+\sum_{j=1}^{n_{\mathrm{enr}}} \mathbf{N}_{j}(\mathbf{x}) \varphi(\mathbf{x}) a_{j},
$$

where $n_{\mathrm{enr}}$ is the number of enriched nodes, $a_{j}$ is the additional contribution for enriched node $j, \mathbf{N}_{j}(\mathbf{x})$ is a shape function from a basis satisfying the partition of unity (classical finite element shape functions are usually considered). The function $\varphi(\mathbf{x})$ represents the enrichment function, which complements the polynomial approximation to represent some complex behaviour such as a discontinuity in the element.

Note that with X-FEM approach, only the nodes belonging to elements containing the interface are enriched. The domain solved by the X-FEM is therefore formed of standard elements, enriched elements, and so-called blending elements as shown in Fig. 2. The elements intersecting the interface are fully enriched (in deep green) while the elements partially enriched are called blending elements (in light green).

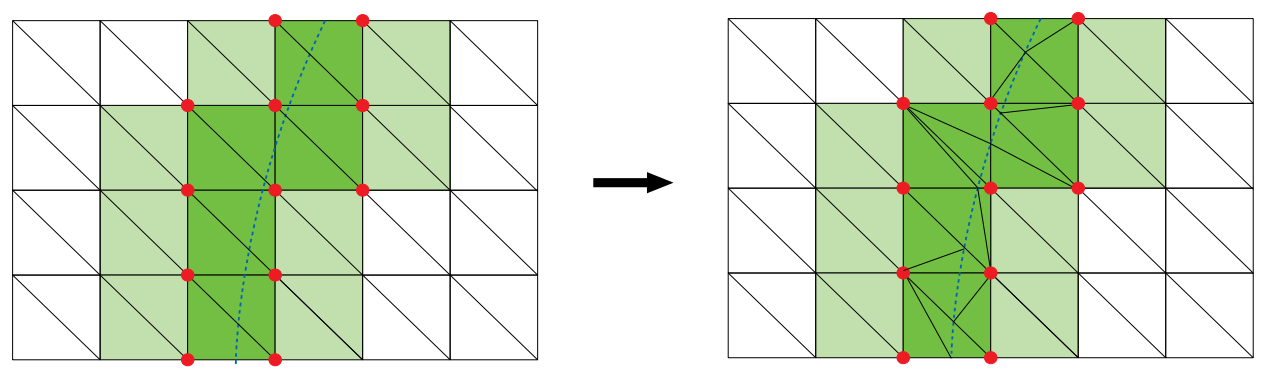

Figure 2: Typical X-FEM mesh with interface. Red nodes: enriched nodes. Deep green: enriched elements. Light green: blending elements. White elements: standard finite elements.

When solving material interface problems with X-FEM, enriched elements at the interface contain different materials (at least two) and the enrichment function is usually continuous at the interface. The enriched elements are partitioned into sub-elements as shown in Fig. 2 (right) to facilitate the numerical integration when computing the algebraic system (details of thus integration method can be found in [21]).

The next ingredient for X-FEM is to track the position of the interfaces and to construct the enrichment function within each enriched element. A level-set function $\phi(\mathbf{x})$ as Eq.(7) is introduced to locate the interface. The function is interpolated by the same finite-element shape functions $N_{i}(\mathbf{x})$ based on the signed distance $\phi_{i}$ of node $i$ to the interface. Nodes are located at one side of 
the interface with $\phi_{i}<0$ while they are located at another side of the interface with $\phi_{i}>0$. A 1D example is shown in Fig. 3a.

$$
\phi(\mathbf{x})=\sum_{i} \phi_{i} N_{i}(\mathbf{x}) .
$$

The approximated location of the interface is given by the zero value of the level-set function: $\phi(\mathbf{x})=0$.

Furthermore, the enrichment function is constructed from the level-set. Since the material interface is a weak discontinuity, a continuous function with a discontinuous derivative defined over the element. It is the so-called "ridge" function proposed in [22]:

$$
R(\mathbf{x})=\sum_{i}\left|\phi_{i}\right| N_{i}(\mathbf{x})-\left|\sum_{i} \phi_{i} N_{i}(\mathbf{x})\right|
$$

where $\phi_{i}$ is nodal signed distance to the interface and $N_{i}(x)$ is the shape function used to approximate the level-set function. The different terms involved in the definition of the enrichment function $R(x)$ are illustrated in 1D in Fig. 3a, Fig. 3b is an illustration of the ridge function in 2D. The ridge enrichment function vanishes on the non-enriched and blending elements, which leads to a good conditioning of the matrix system 22, contrary to other enrichment functions proposed in [21, 30].

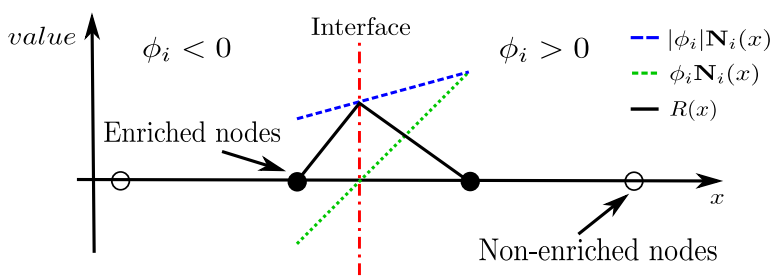

(a)

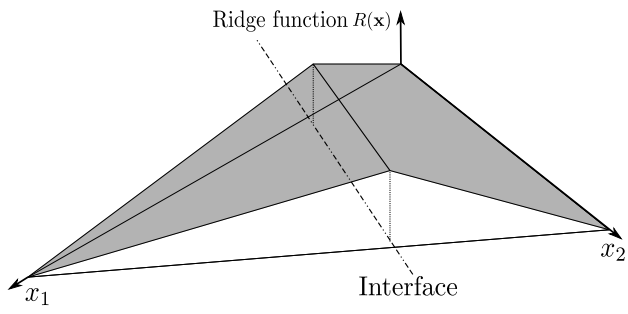

(b)

Figure 3: Level set and ridge enrichment function (a) Definition in 1D, (b) Illustration on a 2D triangular element

If the mesh conforms to the interface profile, the X-FEM recovers the classical FEM since no node is enriched. Note that the ridge enrichment function constructed by a linear approximation of the level-set function may suffer from convergence issues when the geometry of the interface is curved, as geometrical error becomes more important than the solution approximation. Alternative methods were proposed in [23, 24] to address this aspect.

\subsection{Applications to interfaces between porous materials}

The Biot mixed formulation introduced in Section 2 involves two different fields: a scalar pressure

field $p$ and a vector displacement field $\mathbf{u}^{s}$. In contrast, the acoustic fluid medium only requires the scalar pressure field. For this reason it is easier to couple two regions with poro-elastic media since the discretization is the same everywhere in the computational domain. The case where a region of acoustic fluid is coupled to a poro-elastic region requires more care and is addressed subsequently in Section 3.2.2. 


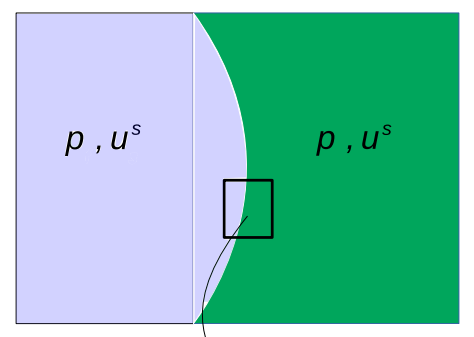

- Standard displacement node

Enriched displacement node

Standard pressure node

Enriched pressure node

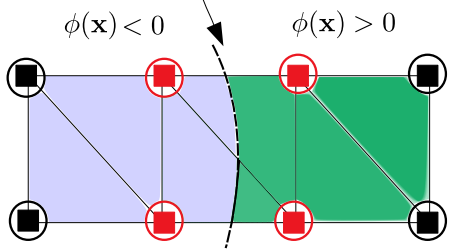

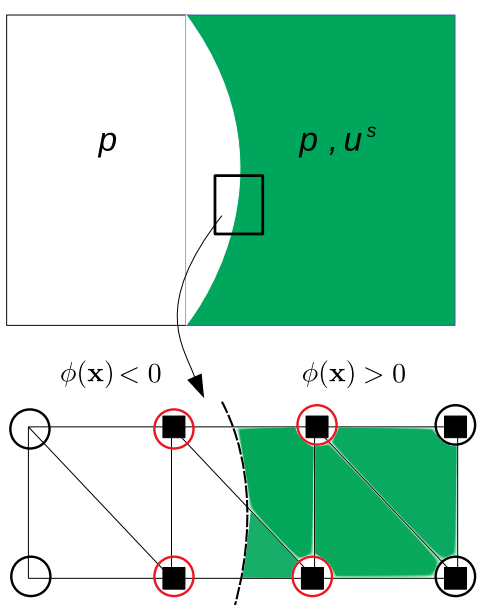

Figure 4: Discretization strategy and node distribution for coupling configuration: PEM-PEM coupling (left) and acoustic fluid-PEM coupling (right).

\subsubsection{Interface between two poro-elastic media}

We first present the interface between two different poro-elastic media modelled with the Biot formulation. Fig. 4 (left) gives an illustration of the discretization strategy for this situation. The whole domain is divided into two subdomains by the interface in order to assign the different material properties in each subdomain. This subdivision is accomplished thanks to the sign of the level-set function $\phi(\mathbf{x})$. The pressure and displacement fields are herein both defined on the whole discretization domain, each node involves three degrees of freedom. The displacement of the solid phase $\mathbf{u}^{s}$ and the pressure $p$ defined on an element $\Omega_{I}$ intersecting the interface are both enriched as follows:

$$
\begin{aligned}
\left.p(\mathbf{x})\right|_{\Omega_{I}} & =\sum_{i}^{n} N_{i}(\mathbf{x}) p_{i}+\sum_{j}^{n_{\text {enr }}} N_{j}(\mathbf{x}) R(\mathbf{x}) a_{j}, \\
\left.\mathbf{u}^{s}(\mathbf{x})\right|_{\Omega_{I}} & =\sum_{i}^{n} \mathbf{N}_{i}(\mathbf{x}) u_{i}^{s}+\sum_{j}^{n} \mathbf{N}_{j}(\mathbf{x}) R(\mathbf{x}) b_{j},
\end{aligned}
$$

where $a_{j}$ and $b_{j}$ are the additional enrichment dofs for the pressure and displacement fields associated to the ridge enrichment function $R(x)$ introduced previously. The conventional shape function $N_{i}(\mathbf{x})$ and $N_{j}(\mathbf{x})$ used in this paper are high order Bernstein basis polynomials. The fields on the elements not containing the interface boundary are expressed by the standard approximation without enrichment terms.

Substituting the prescribed approximations into the weak formulation Eq.(1) and the assembly of element contributions result in a linear system of the form

$$
\left[\begin{array}{cc|cc}
\frac{1}{\omega^{2} \tilde{\rho}_{e q}}\left[\mathbf{K}_{p}\right]-\frac{1}{\tilde{K}_{e q}}\left[\mathbf{M}_{p}\right] & -\tilde{\gamma}\left[\mathbf{C}_{p u}\right] & \frac{1}{\omega^{2} \tilde{\rho}_{e q}}\left[\mathbf{K}_{p a}\right]-\frac{1}{\tilde{K}_{e q}}\left[\mathbf{M}_{p a}\right] & -\tilde{\gamma}\left[\mathbf{C}_{p b}\right] \\
-\tilde{\gamma}\left[\mathbf{C}_{u p}\right] & \hat{P}\left[\mathbf{K}_{u}\right]-\omega^{2} \tilde{\rho}\left[\mathbf{M}_{u}\right] & -\tilde{\gamma}\left[\mathbf{C}_{u a}\right] & \hat{P}\left[\mathbf{K}_{u b}\right]-\omega^{2} \tilde{\rho}\left[\mathbf{M}_{u b}\right] \\
\hline \frac{1}{\omega^{2} \tilde{\rho}_{e q}}\left[\mathbf{K}_{a p}\right]-\frac{1}{\tilde{K}_{e q}}\left[\mathbf{M}_{a p}\right] & -\tilde{\gamma}\left[\mathbf{C}_{a u}\right] & \frac{1}{\omega^{2} \tilde{\rho}_{e q}}\left[\mathbf{K}_{a}\right]-\frac{1}{\tilde{K}_{e q}}\left[\mathbf{M}_{a}\right] & -\tilde{\gamma}\left[\mathbf{C}_{a b}\right] \\
-\tilde{\gamma}\left[\mathbf{C}_{b p}\right] & \hat{P}\left[\mathbf{K}_{b u}\right]-\omega^{2} \tilde{\rho}\left[\mathbf{M}_{b u}\right] & -\tilde{\gamma}\left[\mathbf{C}_{b a}\right] & \hat{P}\left[\mathbf{K}_{b}\right]-\omega^{2} \tilde{\rho}\left[\mathbf{M}_{b}\right]
\end{array}\right]\left\{\begin{array}{c}
\mathbf{p} \\
\mathbf{\mathbf { u } ^ { s }} \\
\frac{\mathbf{a}}{\mathbf{b}}
\end{array}\right\}=\left\{\begin{array}{c}
\mathbf{F}_{p} \\
\mathbf{F}_{u} \\
\mathbf{F}_{e} \\
\mathbf{F}_{e}
\end{array}\right\}
$$


where $\left[\mathbf{K}_{u}\right]$ and $\left[\mathbf{M}_{u}\right]$ are the standard global stiffness and mass matrices for the solid phase, while $\left[\mathbf{K}_{p}\right]$ and $\left[\mathbf{M}_{p}\right]$ are the kinetic and compression energy matrices of the fluid phases. The coupling terms $[\mathbf{C}]$ corresponds to the inner products of shape functions and their derivatives. The matrices with subscript $a$ and $b$ are associated to the enriched terms, i.e. those including the ridge function $R(x) . \mathbf{F}_{p}$ is the external excitation on the fluid phase and $\mathbf{F}_{u}$ represents the external forces on the solid phase. $\mathbf{F}_{e}$ represents the external force applied on the enriched elements. The upper-left block of above the system represents the classical discretized form of the formulation Eq.(1) in the standard finite element method as given in [31], the enrichment method is involved in the remaining terms of the system.

It is worth reminding that each term in the enriched blocks is integrated using a modified Gauss quadrature scheme for partitioned sub-elements at the interface as described previously (see Fig. 2).

\subsubsection{Interface between an acoustic fluid and a poro-elastic medium}

Contrary to the previous configuration, the coupling of an acoustic fluid with a poro-elastic medium involves two different constitutive models: the acoustic fluid is described by Eq.(2) for the pressure field while Eq.(1) with both pressure and displacement describes the poro-elastic medium. The discretization strategy in this situation has to include the following features:

- Assignment of different materials properties as in section 3.2.1.

- Selection of the PEM sub-domain to define the displacement field associated to polynomial spaces;

- Render different governing formulations for acoustic fluid part and porous material part respectively.

The assignment of material properties is handled in the same way as with the PEM-PEM interface, hrough the subdivision of the whole computational domain. Thanks to the interface defined by the level-set function, the displacement field is defined only on the elements on which the level-set function $\phi(\mathbf{x})>0$. The third feature is realized by decomposing the variational formulation into different computational domains. As the pressure field is continuous between the two media, the Helmholtz bilinear forms in Eq.(2) and Eq. (1b) for the pressure terms are defined on the whole domain. The coupling terms with $\tilde{\gamma}$ and the solid dynamic terms in Eq.(1a) are defined on the poro-elastic sub-domain only.

The pressure and the displacement fields are both defined on the elements intersecting the interface to ensure dofs consistency as shown in Fig. 4 (right). However, the displacement is not taken into account on the acoustic fluid side at the numerical integration step. The pressure field approximation on the elements crossed by the interface is enriched, whereas the displacement does not need to be enriched and is approximated with the standard interpolation:

$$
\begin{gathered}
\left.p(\mathbf{x})\right|_{\Omega_{I}}=\sum_{i}^{n} N_{i}(\mathbf{x}) p_{i}+\sum_{j}^{n_{\mathrm{enr}}} N_{j}(\mathbf{x}) R(\mathbf{x}) a_{j}, \\
\left.\mathbf{u}^{s}(\mathbf{x})\right|_{\Omega_{I}}=\sum_{i}^{n} \mathbf{N}_{i}(\mathbf{x}) u_{i} .
\end{gathered}
$$

As before, the fields on the other elements are interpolated by the standard approximation without any enrichment. With these approximations, coupling the Eq.(2) and Eq.(1) leads to the following 
linear system for acoustic fluid-PEM interface problem:

$$
\left[\begin{array}{ccc|c}
\frac{1}{\omega^{2} \rho}\left[\mathbf{K}_{p}\right]-\frac{1}{K}\left[\mathbf{M}_{p}\right] & \mathbf{0} & \mathbf{0} & \frac{1}{\omega^{2} \rho}\left[\mathbf{K}_{p a}\right]-\frac{1}{K}\left[\mathbf{M}_{p a}\right] \\
\mathbf{0} & \frac{1}{\omega^{2} \rho}\left[\mathbf{K}_{p}\right]-\frac{1}{K}\left[\mathbf{M}_{p}\right] & -\tilde{\gamma}\left[\mathbf{C}_{p u}\right] & \frac{1}{\omega^{2} \rho}\left[\mathbf{K}_{p a}\right]-\frac{1}{K}\left[\mathbf{M}_{p a}\right] \\
\mathbf{0} & -\tilde{\gamma}\left[\mathbf{C}_{u p}\right] & \hat{A}\left[\mathbf{K}_{u}\right]-\omega^{2} \rho\left[\mathbf{M}_{u}\right] & -\tilde{\gamma}\left[\mathbf{C}_{u a}\right] \\
\hline \frac{1}{\omega^{2} \rho}\left[\mathbf{K}_{a p}\right]-\frac{1}{K}\left[\mathbf{M}_{a p}\right] \frac{1}{\omega^{2} \rho}\left[\mathbf{K}_{p a}\right]-\frac{1}{K}\left[\mathbf{M}_{p a}\right] & -\tilde{\gamma}\left[\mathbf{C}_{a u}\right] & \frac{1}{\omega^{2} \rho}\left[\mathbf{K}_{a}\right]-\frac{1}{K}\left[\mathbf{M}_{a}\right]
\end{array}\right]\left\{\begin{array}{c}
\mathbf{p}_{a} \\
\mathbf{p} \\
\frac{\mathbf{u}^{s}}{\mathbf{a}}
\end{array}\right\}=\left\{\begin{array}{c}
\mathbf{F}_{a} \\
\mathbf{F}_{p} \\
\mathbf{F}_{u} \\
\mathbf{F}_{e}
\end{array}\right\}
$$

where the unknown $\mathbf{p}_{a}$ represents the pressure field in the acoustic fluid and $\mathbf{F}_{a}$ represents the external excitation in this sub-domain. As for the matrix in Eq.(10), the upper-left block represents the classical FEM system, the same matrix can be found in 32. The last row and column in the system correspond to the contribution of the enrichment terms associated to the additional degrees of the freedom a to maintain the continuity of pressure. Again, the numerical quadrature on each enriched term in the matrix is conducted on the sub-elements associated to the corresponding material type.

It is worth emphasizing that the additional degrees of freedom $a$ and $b$ are added locally on the elements containing the interface but not for all the elements. As a consequence, the linear system to be solved does not become much larger than the system obtained from the classical finite element method. The above discretization strategies based on the X-FEM allow to represent the interface in the elements for different coupling types, but the computation procedure is still based on the idea of the classical FEM. Therefore, some typical characteristics of the solution shall be conserved in the present method when the problem is solved with the classical FEM.

\section{Numerical examples}

In this section, the proposed method is assessed using several numerical examples with increasing geometric complexity of the interface and involving various materials. For the examples with exact solutions, an analysis of convergence is performed.

In this paper, the convergence is measured through the relative error in the $L^{2}$ norm defined for complex-valued fields as follows

$$
\varepsilon_{L^{2}}=\frac{\left\|f_{\mathrm{ex}}-f_{\mathrm{FE}}\right\|_{L^{2}}}{\left\|f_{\mathrm{ex}}\right\|_{L^{2}}} \times 100 \% \text {, with }\|f\|_{L^{2}}=\left(\int_{\Omega}|f|^{2} d \Omega\right)^{1 / 2} .
$$

The errors on the solid phase displacement $\mathbf{u}^{s}$ and on the fluid phase pressure $p$ are calculated separately in order to distinguish the different convergence behaviour for each field. In acoustic problems, the dispersion error or the pollution effect plays an important role on the convergence behaviour. Dispersion error comes from the difference between the theoretical wave number and numerical wave number represented by the computational scheme. A sufficient amount of dofs per wavelength is needed to control this error. The number of dofs per wavelength $D_{\lambda}$ is defined:

$$
D_{\lambda}=\frac{2 \pi \mathrm{P}}{k h}
$$

where $\mathrm{P}$ is the interpolation degree of the shape function and $h$ represents the element size. The Biot equations for the PEM in 2D supports three distinct complex-valued wave numbers. To emphasize the upper limit of the convergence condition, the largest absolute value of the three wave numbers is used:

$$
k^{*}=\left\{\left|k_{i}\right|\right\}_{\max }, \quad \text { with } i=1,2,3
$$


and for two-dimensional problems it is customary to use the total number of degrees of freedom $N_{\text {dof }}$ and the area of the computational domain area $(\Omega)$ instead of $\mathrm{P}$ and $h$ to define an average number of dofs per element as in 33 . Hence the $D_{\lambda}$ is rewritten as:

$$
D_{\lambda}=\frac{2 \pi}{k^{*}} \sqrt{\frac{N_{\text {dof }}}{\operatorname{area}(\Omega)}},
$$

The mesh resolution for the following convergence analysis is measured by $D_{\lambda}$ instead of mesh size $h$ as in classical FEM, which allows to compare the quality of the $h$-extension, polynomial order P-refinement and enriched FEM with the same metric.

Three different porous materials are considered in this paper. These materials are already used in earlier studies[15, 9, 16. The properties of the PEM are listed in Appendix A Table A.1. the first material is a well-characterized foam, the second one represents a polyurethane foam with open-cell structure and whose thermal characteristic length is much higher than common foams while the XFM is a frequently used material in the automotive industry.

The variation of wave numbers as a function of frequency is illustrated in Fig. 5. Three types of waves are present in a PEM: a compression and a shear wave for the vibration of the solid phase, and the compression wave in the fluid phase. For the three materials, there is a frequency where an intersection of two wave numbers is observed. When this occurs, there is a strong coupling between the fluid and solid phases. As frequency increases, the wavenumbers of the solid-borne waves increase faster than that of the fluid wave. The waves then reach a decoupling region where the gap between the wave numbers is large. The solution and the behaviour of the convergence is directly related to these variations of the wave numbers, which will be discussed in the following numerical tests. 


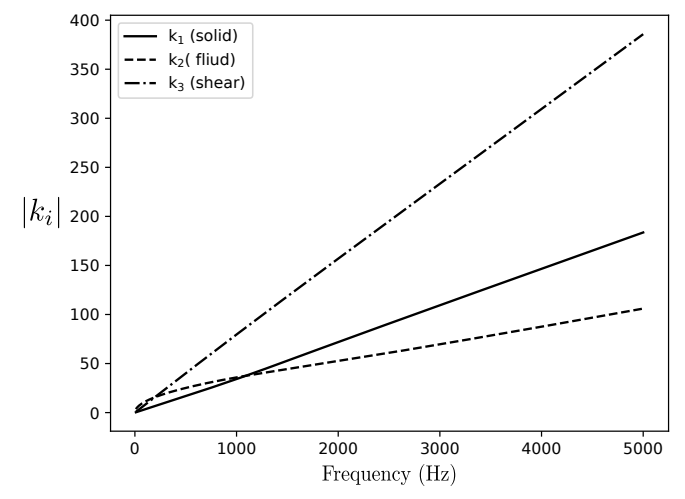

(a)

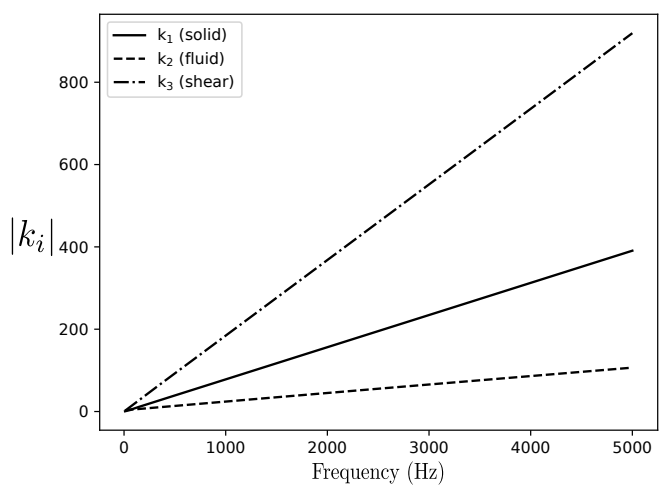

(b)

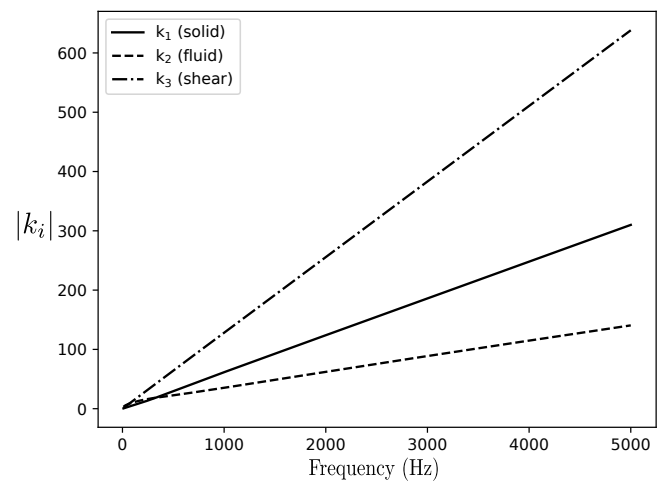

(c)

Figure 5: Wave numbers of the three PEM waves as functions of frequency for (a) a classical foam, (b) polyurethane and (c) XFM (obtained from Appendix A).

\subsection{Plane wave propagating in a double-layered square}

A standard benchmark is first considered, where an oblique plane wave propagates in a semi-infinite plane filled with an acoustic fluid. This example can be regarded as a simplification of a multilayer problem (where number of layer is greater than 2) in practical applications. To test the two coupling conditions (acoustic fluid-PEM and PEM-PEM), two independent configurations are analysed, as shown in Fig. 6. In the first type of coupling, air is considered as the acoustic fluid, the polyurethane is used as the material for the poro-elastic region. The chosen foam is attached on the polyurethane as the second layer in the PEM-PEM coupling.

The exact solutions associated to these configurations are obtained by calculating the reflection $R_{i}$ and transmission $T_{i}$ coefficients with coupling conditions at the interface as shown in Fig. 6. This involves solving $4 \times 4$ or $6 \times 6$ linear systems depending on the type of coupling conditions. The detailed procedure can be found in [28]. 


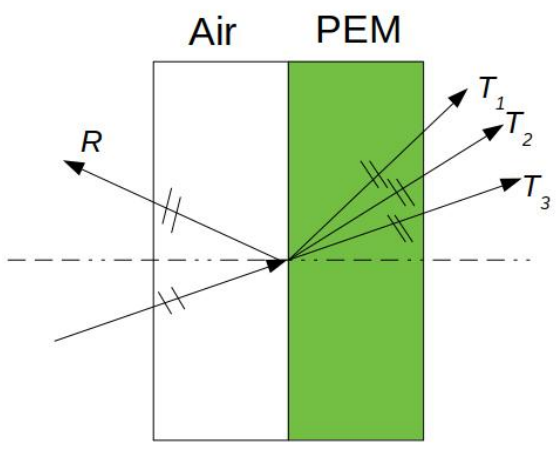

(a)

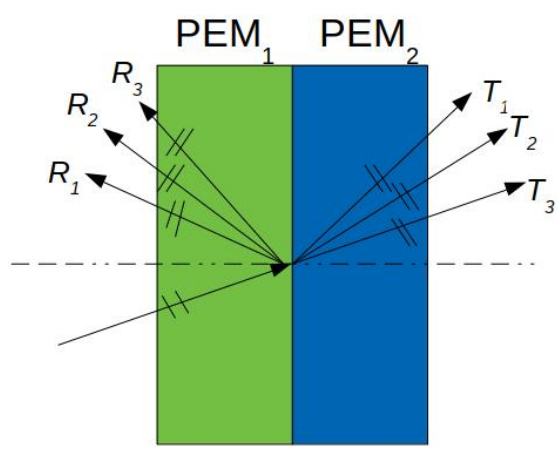

(b)

Figure 6: Test configurations (a) Air-PEM coupling, (b) PEM-PEM coupling.

The computational domain is a square of side $L(0.2 \mathrm{~m}$ for air-PEM and $0.1 \mathrm{~m}$ for the PEMPEM case) discretized by structured triangular elements. The arbitrary medium interface defined by a level-set function is placed at $x=0$ in order to ease the formulation of the exact solution. Exact Neumann boundary conditions are imposed on the four outer boundaries: For the Air-PEM coupling, fluid velocity in air $v_{a}$ are imposed on boundaries of the acoustic fluid media (green line), total displacement $\mathbf{u}^{t}$ and in-vacuo stress $\hat{\sigma}^{s}$ are imposed on the boundaries of the poro-elastic media part (blue line) as shown in Fig. 7a for the two PEMs coupling, total displacement $\mathbf{u}^{t}$ and in-vacuo stress $\hat{\sigma}^{s}$ are imposed on all outer boundaries (blue line) as in Fig. 7b. The simulations are performed from low frequencies in the coupling region to relatively high frequencies in the decoupling region, which allows us to identify different behaviours of solutions and convergence curves depending on the frequency.

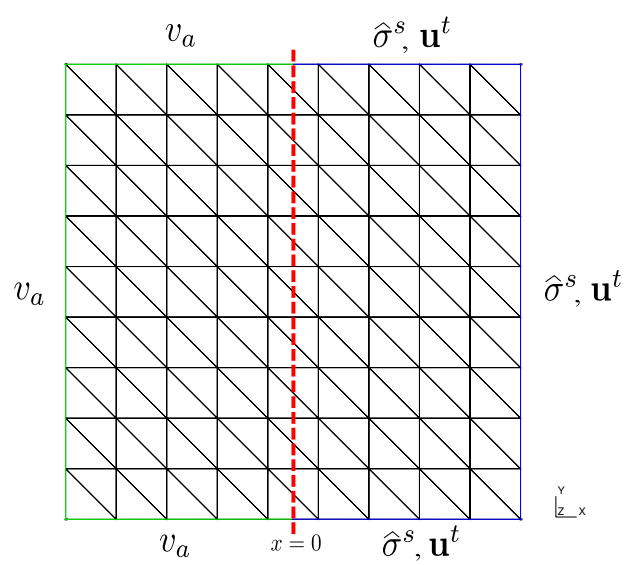

(a)

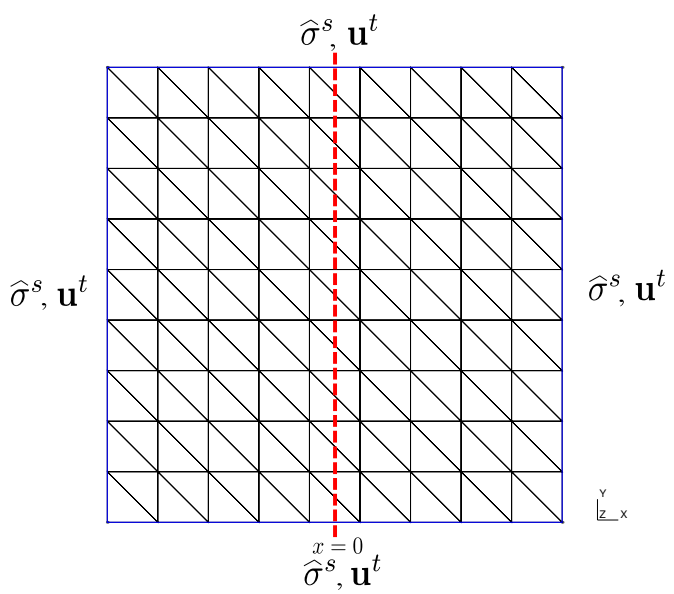

(b)

Figure 7: Example of X-FEM meshes and imposed boundary conditions for (a) Air-PEM coupling,(b) PEM-PEM coupling.

It is recognized firstly from Fig. 8 that the interface $(x=0)$ is embedded in elements instead of aligning with the boundaries of the elements. A change of propagation angle is observed at $x=0$ (illustrating the change of material properties) is successfully handled in the elements by the proposed method. Such a complex oscillation of the waves is well captured using such a relatively coarse mesh thanks to the high polynomial orders of the finite-element basis (Forth order in the 
example presented in Fig. 8).

Figs. 8a and 8b show the real part of the solution for pressure and the $x$ component of the solid displacement at $5,000 \mathrm{~Hz}$ with incident angle of $\pi / 4$ in air-PEM coupling configuration. A dissipation of the pressure wave is observed in the sub-domain $x>0$. The solution for the displacement is only computed for $x>0$ as shown in Fig. 8b since it only exists in the porous material. Figs. 8c and $8 \mathrm{~d}$ illustrate the solution of the PEM-PEM coupling configuration at $2,000 \mathrm{~Hz}$ with incident angle $\pi / 18$. A rapid attenuation for both pressure and displacement on the whole computational domain and the changes of the wave angle at $x=0$ are observed. Note that a great disparity of the scale $\left(>10^{7}\right)$ between displacement and pressure is visible at these frequencies, which is one of the reasons that restricts the convergence of the Biot coupled formulation as discussed in [8].

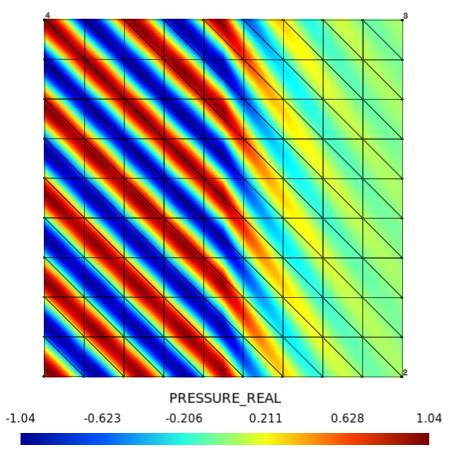

(a)

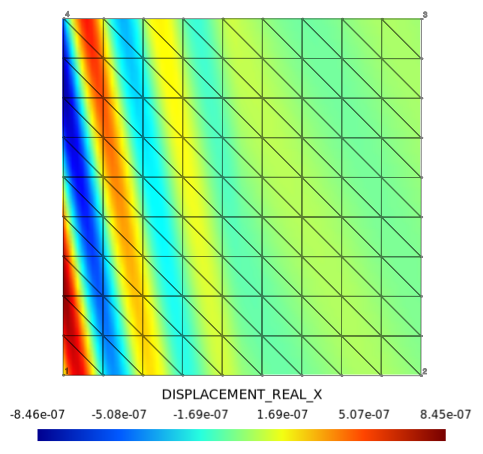

(d)

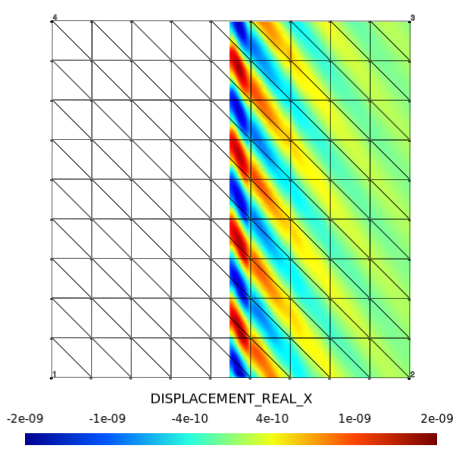

(b)

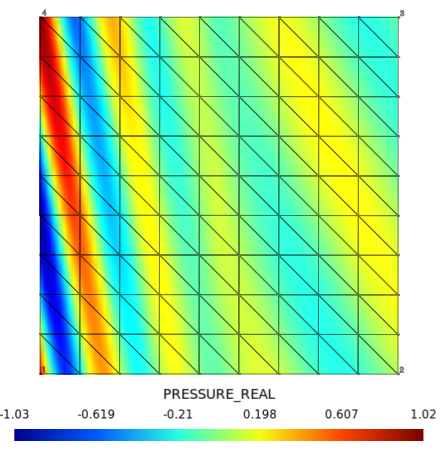

(c)

Figure 8: Example of solution (real part) of Air-PEM coupling (top) with incident angle $\pi / 4$ at $5,000 \mathrm{~Hz}$ and of PEM-PEM coupling (bottom) with incident angle $\pi / 18$ at $2,000 \mathrm{~Hz}$ for fluid pressure (in $\mathrm{Pa}$ ) (a,c) and solid displacement (in $\mathrm{m})(\mathrm{b}, \mathrm{d})$ under fourth interpolation order. 


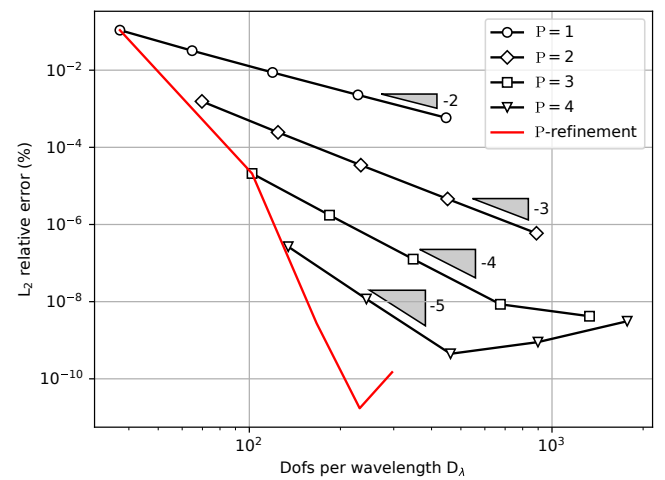

(a)

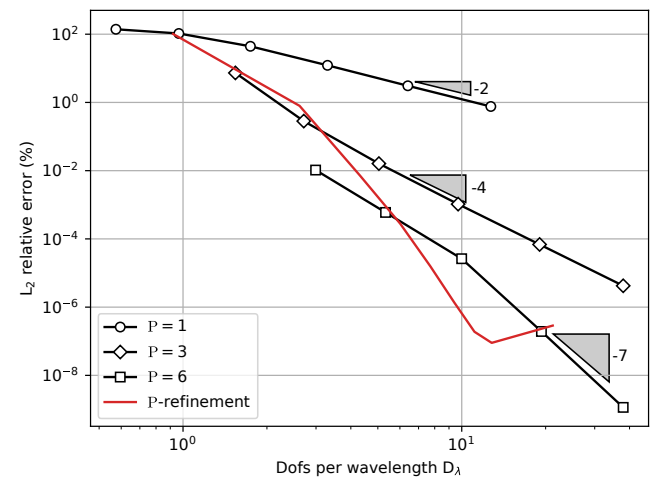

(c)

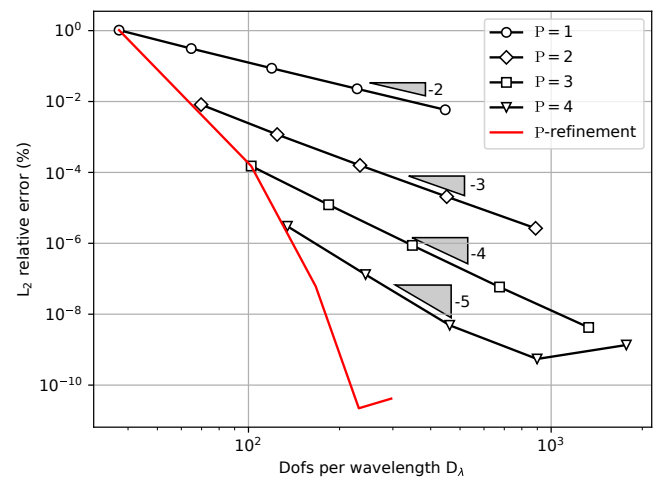

(b)

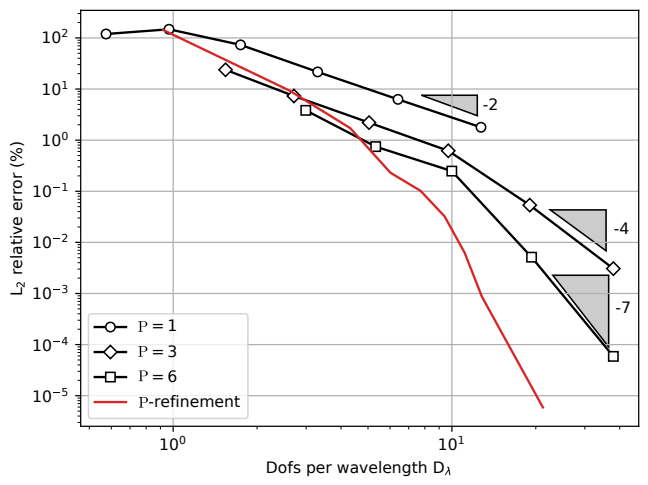

(d)

Figure 9: Relative error $L^{2}$ in percentage on $p$ (left) and $\mathbf{u}^{s}$ (right) as function of $D_{\lambda}$ at $70 \mathrm{~Hz}$ (top) and $5,000 \mathrm{~Hz}$ (bottom) for Air-PEM coupling.

Figs. 9 and 10 shows the convergence in $L^{2}$ norm when refining the element size $h$ and interpolation order $\mathrm{P}$ versus $D_{\lambda}$ carried out for two coupling types and for two frequencies $(70 \mathrm{~Hz}$ and $5,000 \mathrm{~Hz}$ ). Again, pressure and displacement errors are calculated separately. The number of elements $N \times N$ in each $h$-refinement varies from $N=5$ to $N=81$, and the P-refinement is performed with the mesh $N=5$.

$h$-refinement curves in each interpolation degree are close to the so-called 'optimal' rate of convergence $\left(\mathcal{O}\left(h^{\mathrm{p}+1}\right)\right)$ using the present method for both configurations (Air-PEM Fig. 9 and PEM-PEM

Fig. 10). P-refinement (in red) shows an exponential rate of convergence compared to $h$-refinement: (i) keeping the same $D_{\lambda}$, it gives a more accurate solution; (ii) achieving the same magnitude of error, it requires less dofs, meaning less computational effort. These rates of convergence are similar to those reported in [9] and [10, in which the Biot equations are solved using the classical FEM with hierarchical polynomials.

At $70 \mathrm{~Hz}$, pressure and displacement present a synchronous monotonic convergence, reaching the same error magnitude with the same $D_{\lambda}$ as shown in Figs. 9a and 9b. This synchronization corresponds to the fact that we are in a strong coupling frequency range between the fluid and solid phases of the PEM (the wave numbers are close to each other). Differences in the convergence behaviour are obvious at $5,000 \mathrm{~Hz}$ for the Air-PEM configuration in Figs. 9c and 9d, Firstly, the dispersion error for both solutions is observed with linear element and there exists a stage of preasymptotic convergence before achieving the 'optimal' rate as $D_{\lambda}$ is not sufficiently large (inferiors 
to ten dofs per wavelength, which agrees to the conclusion in [8]). Secondly, the pressure field converges more quickly than displacement in $h$-refienement (for the same $D_{\lambda}$, pressure is three orders of magnitude higher than the displacement), which stems from the large difference of wave number at this frequency: the shear solid-borne wave is ten times larger than the compression air-borne wave, as shown in Fig. 5b.

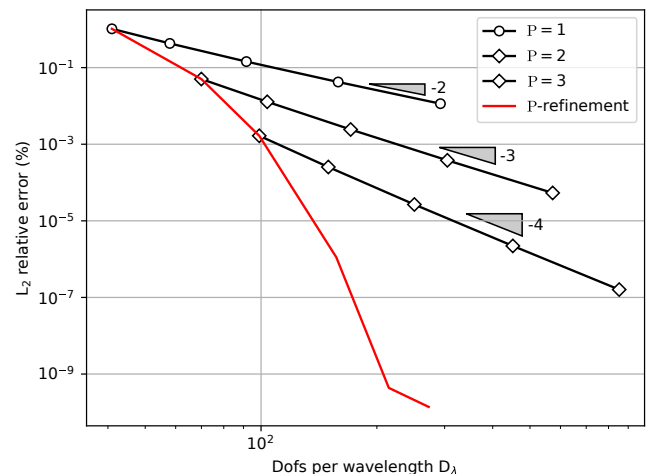

(a)

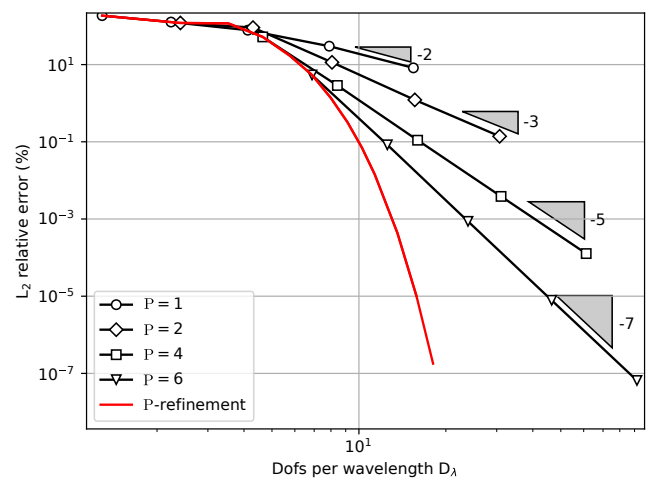

(c)

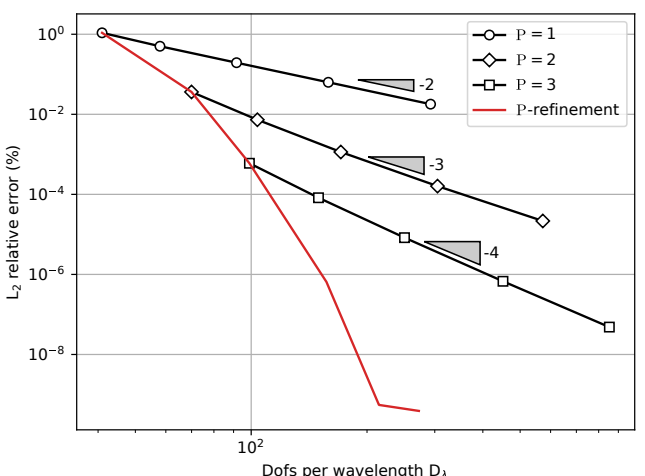

(b)

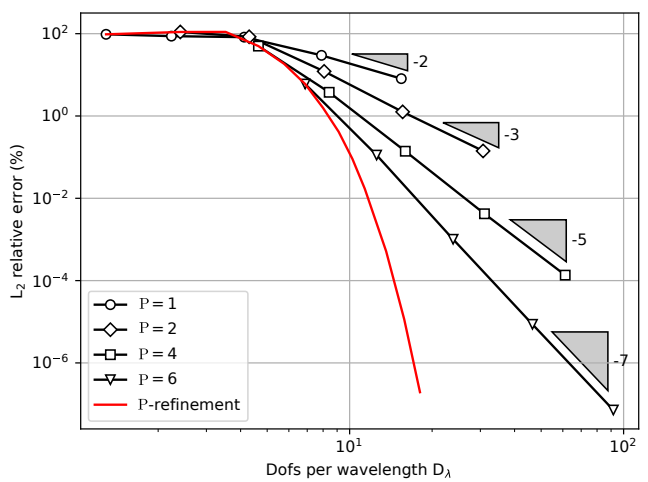

(d)

Figure 10: Relative error $L^{2}$ in percentage on $p$ (left) and $\mathbf{u}^{s}$ (right) as function of $D_{\lambda}$ at $70 \mathrm{~Hz}$ (top) and 5,000 Hz (bottom) for PEM-PEM coupling

Contrary to the Air-PEM coupling, the convergence of the PEM-PEM coupling with chosen materials shows a better concordance between pressure and displacement in Fig. 10. The error magnitudes are of the same order even in the decoupling region (here $5,000 \mathrm{~Hz}$ ). This is because the solutions are close to zero in most region of the computational domain with the double-layer absorbing materials at these frequencies. This configuration is much easier to converge for both pressure and displacement.

Note that in Figs. 9a 9c and Figs. 10a 10b, a stagnation or even an increase of the error at the end of convergence curves is observed. This is due to the fact that the conditioning of the global linear system is poor for these high refinements. This is also reported when using other numerical methods to solve the Biot mixed formulation [15]. This ill-conditioning could be improved with a suitable pre-conditioner [34] or condensation as advocated in [33. Nevertheless, a desirable accuracy is obtained with the present method for this problem. 


\subsection{Sound scattering by a poro-elastic cylinder}

The second test case involves a 2D PEM cylindrical scatterer surrounded by air, thus introducing an acoustic fluid-PEM coupling interface. This case provides an assessment of the proposed method for a more complicated case in both physic and computational aspects. Sound radiation problems in free field involving poro-elastic media result in more complex distributions of the acoustic fields. In addition, the presence of a curved interface may introduce geometrical error in the numerical approximation. An exact solution is also available. It is recalled in Appendix B or can be found in [35.

The computational domain is a square of length of $0.5 \mathrm{~m}$ meshed using structured triangular elements. The exact velocity of the fluid in air $v_{a}$ obtained from the analytical solution is imposed on the outer boundaries (green line) of the domain, as illustrated in Fig. 11a. Within the proposed method, the circular interface with radius $R=0.1 \mathrm{~m}$ is described implicitly by a level-set function using a linear approximation. The porous subd-omain governed by the Biot equations (1) is defined for $r<R$. For higher-order approximations $(\mathrm{P} \geq 2)$, the NURBS enhanced X-FEM strategy [25] is considered to avoid the geometrical error caused by the linear interpolation of the level-set. The circular boundary of the interface is better described using a coarse mesh as shown in Fig. 11b (the global enrichment strategy is detailed in 25]). It is clear that the mesh used in the present method is independent of the circular interface.

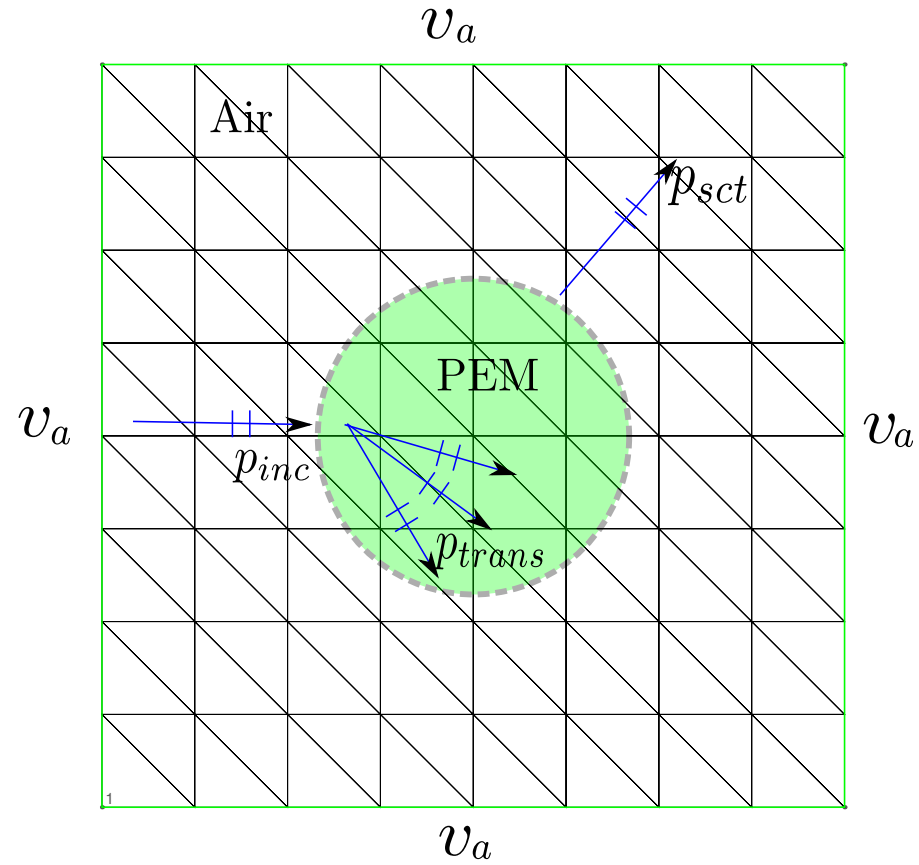

(a)

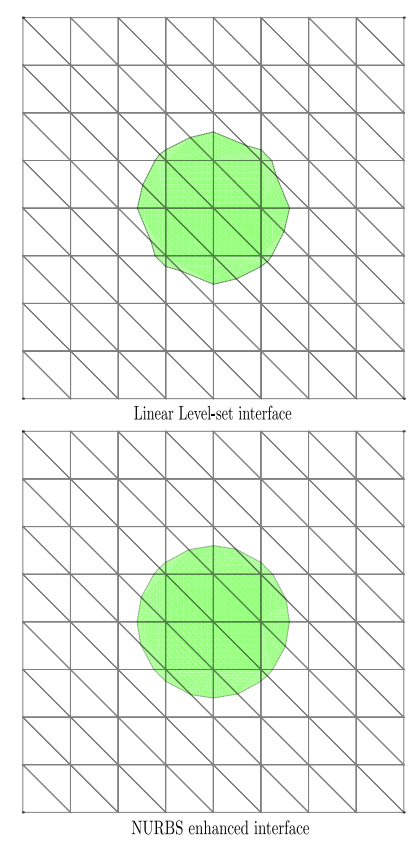

(b)

Figure 11: Cylinder scattering problem with an example of X-FEM mesh (a); Linear Level-set interface and NURBS enhanced interface using same mesh (b).

Results in Fig. 12a give a good illustration of the pressure distribution for the porous scattering problem. The combination of the incident $p_{\text {inc }}$ and the partly reflected waves $p_{\text {sct }}$ is observed in outer sub-domain $r>R$. The waves are partly absorbed by the porous material in the region $r<R$. As seen in the Fig. 12b, the displacement of the solid phase is defined and computed in the absorbing region, whose oscillation and dissipation are also well captured. Again, the accuracy of the present method is measured with the relative error compared to the exact solution in $L^{2}$ norm. The convergences of $h$-refinements are conducted using regular triangular $N \times N$ meshes: 
$N=4,8,16,32,64$ and P-convergence is performed with the mesh $N=4$ with an interpolation 360 order varying between one and fourteen. The convergences at $70 \mathrm{~Hz}$ and $2,000 \mathrm{~Hz}$ are shown in Fig. 13 .

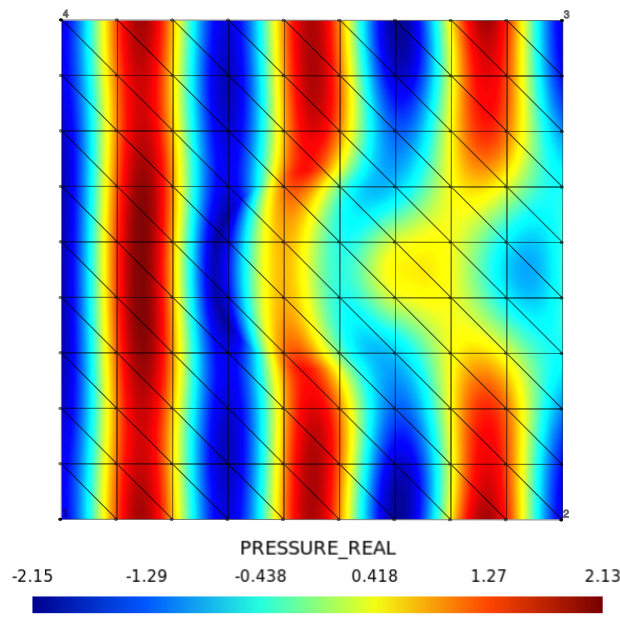

(a)

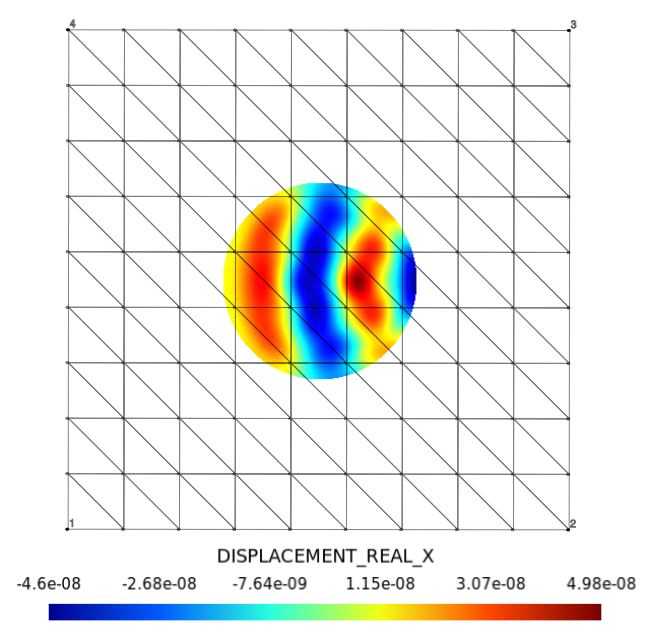

(b)

Figure 12: Example of solution (real part) for (a) pressure (in Pa) and (b) displacement (in $\mathrm{m}$ ) of cylinder scatter problem in $2,000 \mathrm{~Hz}$ with the fourth order polynomials. 


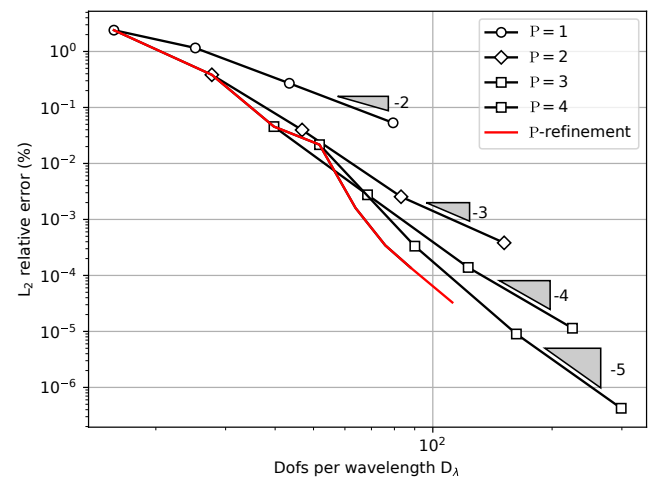

(a)

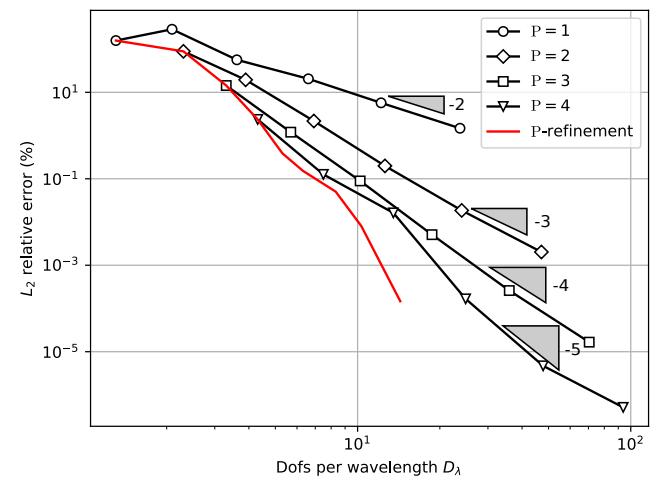

(c)

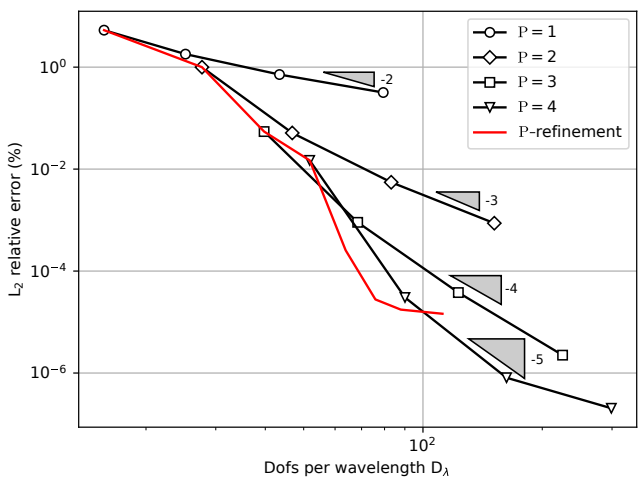

(b)

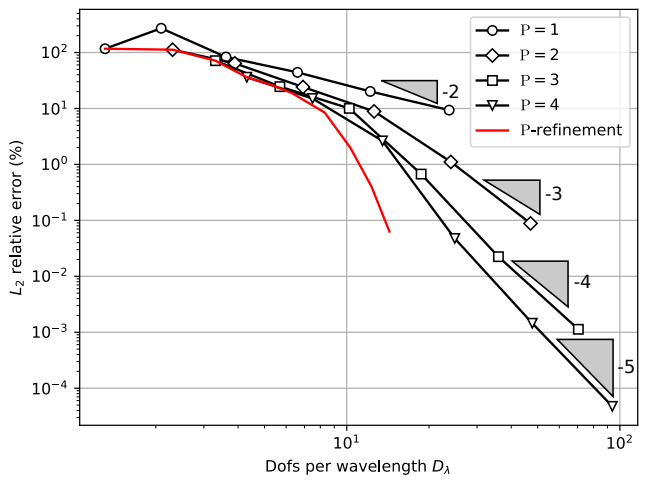

(d)

Figure 13: Relative error $L^{2}$ in percentage on $p$ (left) and $\mathbf{u}^{s}$ (right) as function of $D_{\lambda}$ at $70 \mathrm{~Hz}$ (top) and 2, $000 \mathrm{~Hz}$ (bottom).

The $h$-refinement results are similar to those presented in the first test case: rates close to $\mathcal{O}\left(h^{\mathrm{P}+1}\right)$ are observed for each approximation order when $D_{\lambda}$ is sufficient. The convergence of linear element for the displacement field in Figs. 13b and 13d do not have sufficiently large $D_{\lambda}$. The cut-on phenomenon (explained in [9]) and a pre-asymptotic regime are more apparent in this problem at $2,000 \mathrm{~Hz}$. These high-order convergence rates are in accordance with previous studies reported in [10. This agreement confirms that the X-FEM with the proposed discretization strategies is capable of providing an accurate solution for this more complex problem. The P-refinement curves in Fig. 13 are less efficient than the one in the previous test case. It is due to the fact that with the interface described by NURBS, the enrichment function is defined with Lagrange polynomials instead of the Bernstein basis. It is well-known that the Lagrange polynomials lead to worse conditioning as the interpolation order increases. Even so, a high accuracy of the solutions for this more complex problem is still achieved.

The convergence of the numerical models for the two test cases described so far has been assessed with various porous materials at different frequencies. For brevity, not all these results are shown here, but the same conclusions apply. The convergence behaviour is strictly linked to the materials and frequencies since each term in the finite element matrix is associated to material properties, which are frequency dependent. These differences result in different conditioning of the matrix, leading to different convergence curves and magnitude of error.

The above numerical tests demonstrate that the proposed method is able to recover an optimal 
rate of convergence regardless of frequency, material, coupling interface, geometry and solution. Concerning the Biot formulations, a higher order of approximation or larger $D_{\lambda}$ are recommended for the displacement field in order to achieve a more accurate convergence for the whole solution.

\subsection{Car cavity with poro-elastic seat}

In this last example, the proposed X-FEM approach is applied to a more industrial test case which corresponds to the interior of a car cavity with a driver seat filled with the porous material $\mathrm{XFM}$ and modeled with the Biot $(\mathbf{u}, p)$ formulation. The dimensions of the car are approximately $2.67 \mathrm{~m} \times 1.1 \mathrm{~m}$. An arbitrary normal velocity $(\partial p / \partial n=1)$ is imposed on the front windscreen to generate a sound field at $2,000 \mathrm{~Hz}$. A common situation where the driver's seat is switched from one position to another position is considered here and the sound pressure distribution (in $\mathrm{dB}$ ) inside the car is computed.

The geometry and the mesh for X-FEM are shown in Fig. 14. The profile of the driver seat within the present method is defined implicitly by level-set functions (in blue and red) and the displacement field is only defined within this sub-domain. With this mesh, the definition of the seat profile is almost independent of the seat position (in order to locate this complex interface accurately by linear level-set, the mesh should not be too coarse). With X-FEM, changing the position of the seat can be realised very easily by simply translating the level-set functions instead of generating a new mesh. This is in stark contrast with the classical finite element method which requires two different meshes for two different seat positions.

A standard finite element resolution is performed as a comparison, whose meshes are shown in Fig. 15. It can be seen that the mesh for the classical FEM needs to conform to the interface between the seat and the air in the cavity. In addition, the mesh is refined at the top of the seat where it has multiple small geometrical features. A polynomial order $\mathrm{P}=4$ is used to obtain a converged sound pressure field with these meshes.

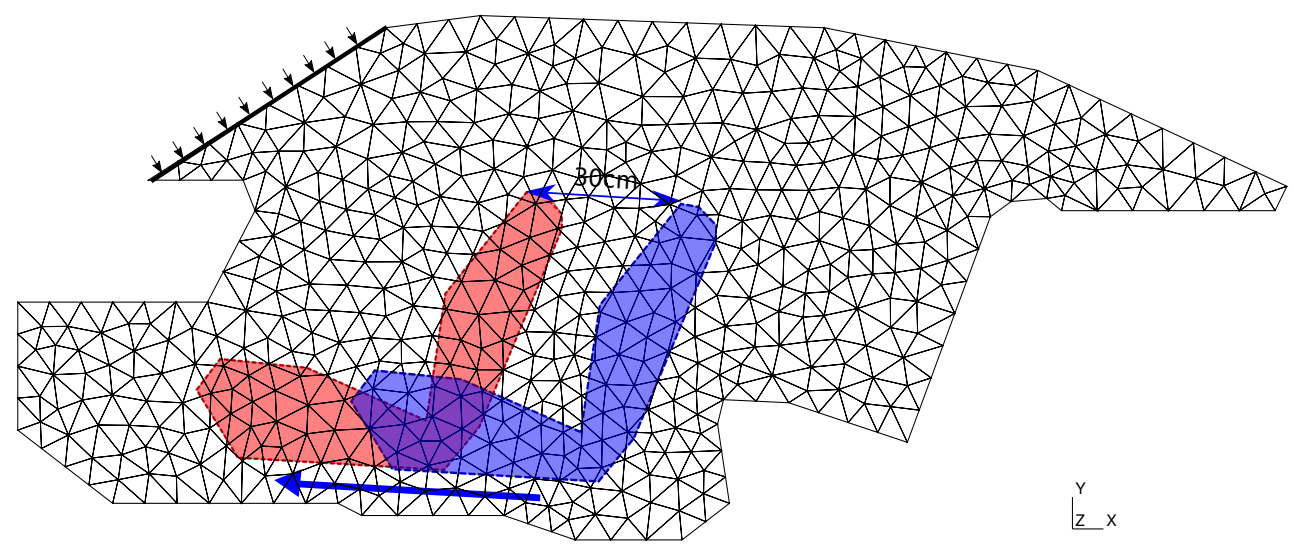

Figure 14: X-FEM mesh for two configurations with the seat defined by level-set functions. 


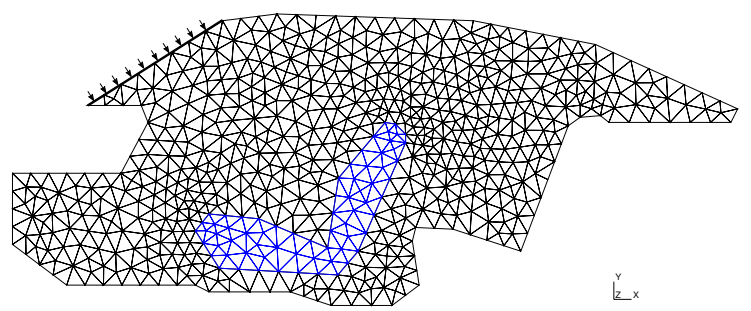

(a)

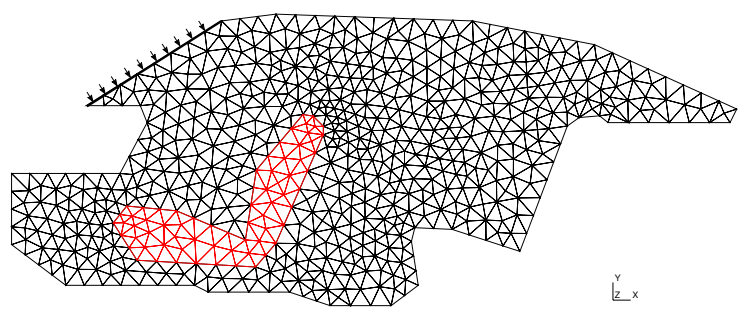

(b)

Figure 15: Standard FEM models with conforming meshes: (a) position A, (b) position B.

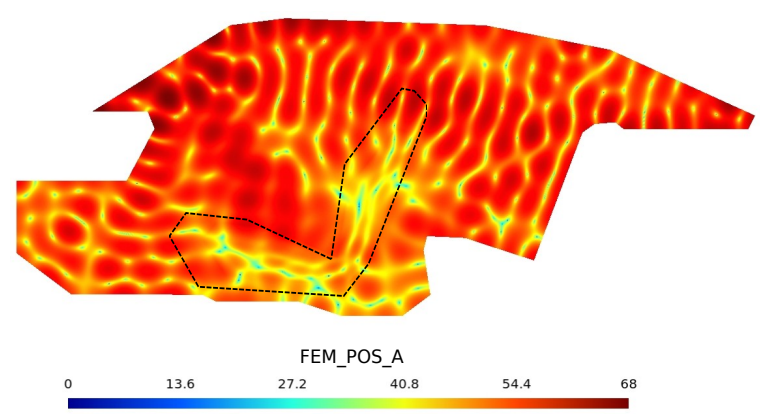

(a)

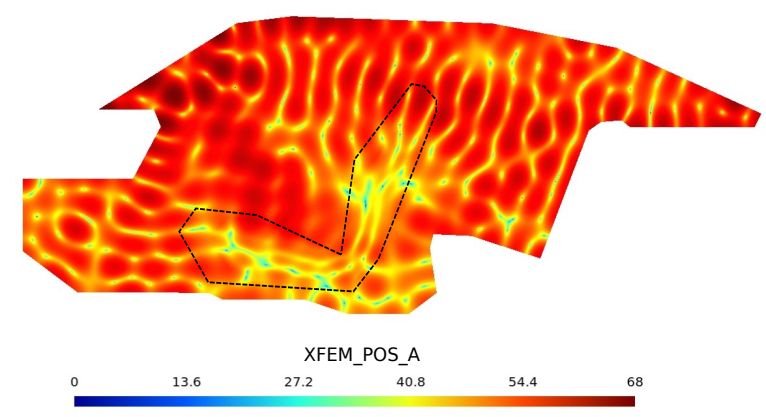

(c)

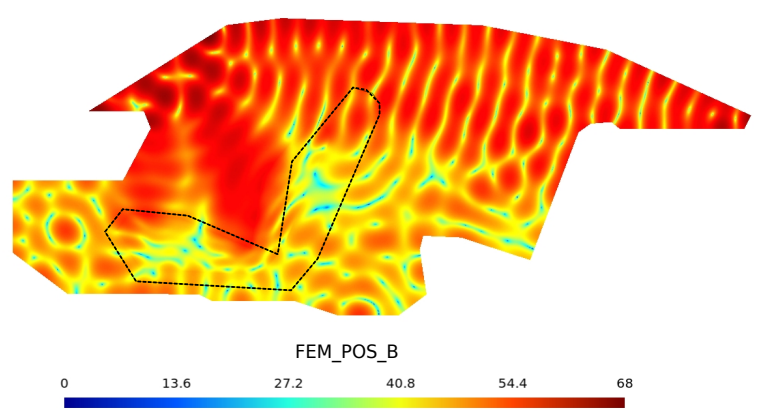

(b)

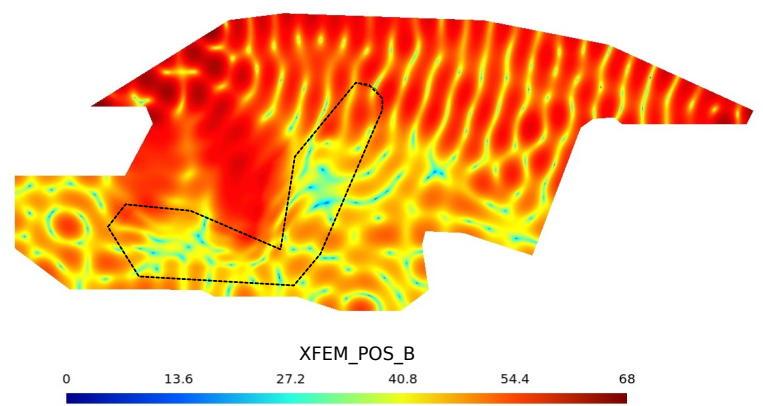

(d)

Figure 16: Solution of the sound pressure (in dB): with classical FEM (top) and X-FEM (bottom) for position A (left) and B (right)

The X-FEM results shown in Figs. 16c and 16d clearly capture the variation of the pressure field as the seat position is changed. The absorption of the sound wave within the seat is also well described. It also shows very good agreement with the classical finite element results shown in Figs. 16a and 16b. Small differences can be observed between the two methods because the meshes used to post-process the results are not strictly identical. This introduces differences in the graphical interpolation. Also, the linear level-set function used to describe the contours of the seat results in minute geometrical errors. This case provides a representative application of the proposed method where transformations such as translation, rotation or symmetry of an internal complex geometry (a porous seat in this case) can be handled without re-meshing. It demonstrates that the method can be a efficient numerical tool for the topological design of absorbing materials in the vibro-acoustics community. 


\section{Conclusion}

This paper investigates the application of the eXtended Finite Element Method (X-FEM) to analyse poro-elastic materials in vibro-acoustics, focusing on modelling an interface between different media. Poro-elastic materials are modelled by a mixed Biot formulation which involves two fields: the pressure $p$ in the fluid phase and the displacement $\mathbf{u}^{s}$ of solid phase.

The discretization strategies based on the X-FEM framework, and on the nature of the Biot formulation, are provided for two types of coupling conditions: acoustic fluid-PEM and PEMPEM. The material interface is defined implicitly by a level-set function, and the field at the interface is enriched with a ridge function to introduce the gradient discontinuity. The accuracy The performance of the method was assessed with different test cases. The solutions for $\mathbf{u}^{s}$ and $p$ are intentionally calculated separately to identify the different convergence behaviours. The two coupling interface conditions are tested for the propagation of a plane waves in a semi-infinite plane. The optimal rates of convergence in the $L^{2}$ norm $\left(\mathcal{O}\left(h^{\mathrm{p}+1}\right)\right)$ are recovered for both fields $\left(\mathbf{u}^{s}, p\right)$ at in this case. A cylindrical scattering problem is also studied. Close-to-optimal convergence rates are recovered, demonstrating that the present method is able to deal with curved interfaces with high approximation order. Finally, a car cavity problem is considered to show the applicability of the present method in an engineering context. The advantages of the proposed method are clearly material can be easily achieved without re-meshing, and the meshes are relatively independent of the interface geometry. These benefits provide a significant computational benefit in engineering situations.

The present work is the first application of a high-order X-FEM framework for solving the Biot equation in the presence of interfaces between different materials. Such problems can now be solved accurately in a wide range of frequencies using a relatively simple mesh that is independent of the geometry. Future work will include the modelling of "imperfect" material interfaces (this will introduce strong discontinuities), as well as more realistic three-dimensional poro-elastic material problems.

\section{CRediT authorship contribution statement}

Shaoqi Wu: Methodology, Software, Formal analysis, Visualisation, Writing-Original draft preparation, Writing-Review\&Editing. Grégory Legrain: Methodology, Software, Writing-Review\&Editing, Supervision. Olivier Dazel: Conceptualization, Validation, Writing-Review\&Editing, Supervision. Gwénaël Gabard: Conceptualization, Validation, Writing-Review\&Editing, Supervision.

\section{Declaration of Competing Interest}

The authors declare that they have no known competing financial interests or personal relationships that could have appeared to influence the work reported in this paper.

\section{Appendix A. Coefficient in Johnson-Champoux and Biot Allard model}

This appendix gives analytical expressions of the coefficients for the Johnson-Champoux model [2 1] and their associated parameters are listed in Table A.1. 
Table A.1: Material parameters

\begin{tabular}{cccc}
\hline Parameters & Foam & Polyurethane & XFM \\
\hline Porosity $\phi$ & 0.98 & 0.98 & 0.98 \\
static flow resistivity $\sigma\left(\mathrm{N} . \mathrm{m}^{-4}\right)$ & $15.5 \times 10^{3}$ & $3.75 \times 10^{3}$ & $13.5 \times 10^{3}$ \\
Tortuosity $\alpha$ & 1.01 & 1.17 & 1.7 \\
thermal characteristic length $\Lambda^{\prime}(\mathrm{m})$ & $250 \times 10^{-6}$ & $742 \times 10^{-6}$ & $160 \times 10^{-6}$ \\
Viscous characteristic length $\Lambda(\mathrm{m})$ & $100 \times 10^{-6}$ & $110 \times 10^{-6}$ & $80 \times 10^{-6}$ \\
frame density $\rho_{1}\left(\mathrm{~kg} . \mathrm{m}^{-3}\right)$ & 11 & 22.1 & 30 \\
Young's modulus $E(\mathrm{~Pa})$ & $200 \times 10^{3}$ & $70 \times 10^{3}$ & $200 \times 10^{3}$ \\
Poisson ratio $\nu$ & 0.35 & 0.39 & 0.35 \\
Loss factor $\eta_{s}$ & 0.1 & 0.265 & 0.05 \\
\hline
\end{tabular}

Effective parameters of equivalent fluid in PEM takes both viscous and thermal effect into consideration. The fluid density $\tilde{\rho}_{e q}$ is written as:

$$
\tilde{\rho}_{e q}=\frac{\rho_{a} \alpha}{\phi}\left(1+\frac{\omega_{0}}{\mathrm{j} \omega} \sqrt{1+\frac{\mathrm{j} \omega}{\omega_{\infty}}}\right)
$$

with $\omega_{0}$ and $\omega_{\infty}$ expressed as:

$$
\begin{gathered}
\omega_{0}=\frac{\sigma \phi}{\rho_{a} \alpha} \\
\omega_{\infty}=\frac{(\sigma \phi \Lambda)^{2}}{4 \eta_{a} \rho_{a} \alpha^{2}}
\end{gathered}
$$

where $\alpha$ is the geometrical tortuosity, $\phi$ is the porosity, $\rho_{a}$ is the interstitial fluid density, $\sigma$ is the flow resistivity, $\eta_{a}$ is the dynamic viscosity of the fluid and $\Lambda$ is the viscous characteristic length. The coupling coefficient $\tilde{\gamma}$ and solid equivalent density are expressed as:

$$
\begin{gathered}
\tilde{\gamma}=\phi\left(\frac{\tilde{\rho}_{12}}{\tilde{\rho}_{22}}-\frac{1-\phi}{\phi}\right) \\
\tilde{\rho}=\tilde{\rho}_{11}-\left(\frac{\tilde{\rho}_{12}^{2}}{\tilde{\rho}_{22}}\right)
\end{gathered}
$$

where $\tilde{\rho}_{11}, \tilde{\rho}_{12}$ and $\tilde{\rho}_{22}$ are the coefficients related to the geometry of the frame, the detailed relationship between $\rho_{a}$ and density of solid phase $\rho_{1}$ are given:

$$
\tilde{\rho}_{22}=\phi^{2} \tilde{\rho}_{e q}, \quad \tilde{\rho}_{12}=\phi \rho_{a}-\tilde{\rho}_{22}, \quad \tilde{\rho}_{11}=\rho_{1}-\tilde{\rho}_{12}
$$

The thermal properties are given by the effective compressibility $\tilde{K}_{e q}$ :

$$
\tilde{K}_{e q}=\gamma p_{0} /\left[\gamma-(\gamma-1) /\left(1+\frac{8 \eta_{a}}{\mathrm{j} \omega \rho_{a} P_{r} \Lambda^{\prime}} \sqrt{1+\frac{\mathrm{j} \omega \rho_{a} P_{r} \Lambda^{\prime 2}}{16 \eta_{a}}}\right)\right]
$$

where $\gamma$ is the ratio of specific heats of air, $p_{0}$ is the referred ambient pressure, $P_{r}$ is the Prandtl number and $\Lambda^{\prime}$ is the thermal characteristic length.

The constitutive relationship between stress in vacuum and strain in solid frame of the porous material is:

$$
\sigma_{i j}^{s}=2 N e_{i j}+\hat{A}\left(e_{k k}\right) \delta_{i j}
$$


where strain $e_{i j}$ is function of the solid displacement:

$$
e_{i j}=\frac{1}{2}\left(\frac{\partial u_{i}^{s}}{\partial x_{j}}+\frac{\partial u_{j}^{s}}{\partial x_{i}}\right)
$$

and the structural lamé coefficient $N$ and shear coefficient are written as:

$$
N=\frac{E\left(1+\mathrm{j} \eta_{s}\right)}{2(1+\nu)}, \quad \hat{A}=\frac{E \nu\left(1+\mathrm{j} \eta_{s}\right)}{(1+\nu)(1-2 \nu)}
$$

and we define:

$$
\hat{P}=\hat{A}+2 N
$$

where $E$ and $\nu$ are the Young's modulus and Poisson coefficient of the elastic solid in vacuum, $\eta_{s}$ is the loss factor of the elastic solid phase with unit imaginary number $\mathrm{j}$.

There exists three waves in materials described by Biot theory. The wave numbers of the two compressional waves are given by solving the eigen-problem of wave equations:

$$
k_{i}^{2}=\frac{\left(k_{s 2}^{2}+k_{e q}^{2}\right) \pm \sqrt{\left(k_{s 2}^{2}+k_{e q}^{2}\right)^{2}-4 k_{e q}^{2} k_{s 1}^{2}}}{2}, i=1,2
$$

with

$$
k_{e q}=\omega \sqrt{\frac{\tilde{\rho}_{e q}}{\tilde{K}_{e q}}}, k_{s 1}=\omega \sqrt{\frac{\tilde{\rho}}{\hat{P}}}, k_{s 2}=\omega \sqrt{\frac{\tilde{\rho}}{\hat{P}}}
$$

The ratio $\mu$ of the total velocity and frame velocity is:

$$
\mu_{i} \tilde{\gamma} \frac{k_{e q}^{2}}{\delta_{i}^{2}-k_{e q}^{2}}, i=1,2
$$

The wave equation for the rotational wave is obtained by using a vector potential as well and the corresponding wave number reads:

$$
k_{3}=\omega \sqrt{\frac{\tilde{\rho}}{N}}
$$

with its ratio between total velocity and frame velocity:

$$
\mu_{3}=-\tilde{\gamma}
$$

\section{Appendix B. Analytical solution for cylinder porous scattering problem}

Plane waves of scattering problem in this paper are written in potential form. Potential of wave

field in acoustic domain with amplitude $A_{i n c}$ is represented in polar coordinate $(r, \theta)$ as:

$$
\varphi=\varphi_{i n c}+\sum_{m=0}^{\infty} \hat{A}_{0, m} \mathrm{H}_{m}\left(k_{a} r\right) \cos (m \theta)
$$

where $\varphi_{i n c}$ is incident wave potential which is propagating along horizontal direction $x$ :

$$
\varphi_{\text {inc }}=A_{\text {inc }} \exp \left(-\mathrm{j} k_{a} x\right)=A_{\text {inc }}\left[\mathrm{J}_{0}\left(k_{0} r\right)+2 \sum_{m=1}^{\infty}(-\mathrm{j})^{m} \mathrm{~J}_{m}\left(k_{a} r\right) \cos (m \theta)\right]
$$


where $\mathrm{H}_{m}(x)$ is the Hankel function of the second kind, $\mathrm{J}_{m}(x)$ is the Bessel function of the first kind. $k_{a}$ represents wave number of air fluid. $\mathrm{j}$ is unit imaginary number with $\mathrm{j}^{2}=-1$.

Potentials of the two compressional waves with amplitude $A_{i}$ in porous material are given:

$$
\varphi_{i}=\sum_{m=0}^{\infty} \hat{A}_{i, m} \mathrm{~J}_{m}\left(k_{i} r\right) \cos (m \theta), \quad \text { with } i=1,2
$$

490

and the one of shear wave in solid phase:

$$
\varphi_{3}=\sum_{m=0}^{\infty} \hat{A}_{3, m} \mathrm{~J}_{m}\left(k_{3} r\right) \sin (m \theta)
$$

where $k_{1}, k_{2}$ and $k_{3}$ are compressional and shear wave numbers in fluid and solid phase respectively in Biot modeled porous material. Pressure in acoustic domain is $p=\rho \omega^{2} \varphi$, while solid displacement in porous material is cast with Helmholtz decomposition as:

$$
\mathbf{u}^{s}=\nabla \varphi+\nabla \wedge \varphi_{3}
$$

where $\varphi=\varphi_{1}+\varphi_{2}$. The other fields such as total displacement $\mathbf{u}^{t}$ and fluid pressure $p$ are obtained by polarization relationship:

$$
\begin{gathered}
\mathbf{u}^{t}=\mu_{i} \mathbf{u}^{s}, \quad \text { with } i=1,2 \\
p=-\tilde{K}_{e q} \nabla \cdot \mathbf{u}^{t}
\end{gathered}
$$

where $\mu_{i}$ is the wave amplitude ratios between solid and total displacement. Once the expressions of all the field are known, the analytical solution is available by solving a $4 \times 4$ linear system with 
the interface conditions Eq.(3) at $r=a$. Each term in the left-hand side matrix is provided:

$$
\begin{aligned}
& M_{1,1}=\frac{m \mathrm{H}_{m}\left(k_{a} a\right)}{a}-k_{a} \mathrm{H}_{m+1}\left(k_{a} a\right) \\
& M_{1,2}=\frac{\mu_{1}\left(-m \mathrm{~J}_{m}\left(k_{1} a\right)\right)+k_{1} \mu_{1} a \mathrm{~J}_{m+1}\left(k_{1} a\right)}{a} \\
& M_{1,3}=\frac{\mu_{2}\left(-m \mathrm{~J}_{m}\left(k_{2} a\right)\right)+k_{2} \mu_{2} a \mathrm{~J}_{m+1}\left(k_{2} a\right)}{a} \\
& M_{1,4}=\frac{-\mu_{3} m \mathrm{~J}_{m}\left(k_{3} a\right)}{a} \\
& M_{2,1}=\rho_{a} \omega^{2} \mathrm{H}_{m}\left(k_{a} a\right) \\
& M_{2,2}=-\tilde{K}_{e q} \mu_{1} k_{1}^{2} \mathrm{~J}_{m}\left(k_{1} a\right) \\
& M_{2,3}=-\tilde{K}_{e q} \mu_{2} k_{2}^{2} \mathrm{~J}_{m}\left(k_{2} a\right) \\
& M_{2,4}=0 \\
& M_{3,1}=0 \\
& M_{3,2}=\frac{2 N}{a^{2}}\left(m(m-1) \mathrm{J}_{m}\left(k_{1} a\right)-k_{1}^{2} a^{2} \mathrm{~J}_{m}\left(k_{1} a\right)+k_{1} a \mathrm{~J}_{m+1}\left(k_{1} a\right)\right)-\hat{A} k_{1}^{2} \mathrm{~J}_{m}\left(k_{1} a\right) \\
& M_{3,3}=\frac{2 N}{a^{2}}\left(m(m-1) \mathrm{J}_{m}\left(k_{2} a\right)-k_{2}^{2} a^{2} \mathrm{~J}_{m}\left(k_{2} a\right)+k_{2} a \mathrm{~J}_{m+1}\left(k_{2} a\right)\right)-\hat{A} k_{2}^{2} \mathrm{~J}_{m}\left(k_{2} a\right) \\
& M_{3,4}=\frac{2 N\left(m(m-1) \mathrm{J}_{m}\left(k_{3} a\right)-k_{3} a m \mathrm{~J}_{m+1}\left(k_{3} a\right)\right)}{a^{2}} \\
& M_{4,1}=0 \\
& M_{4,2}=\frac{2 N k_{1} a m \mathrm{~J}_{m+1}\left(k_{1} a\right)-N m(2 m-2) \mathrm{J}_{m}\left(k_{1} a\right)}{a^{2}} \\
& M_{4,3}=\frac{2 N k_{2} a m \mathrm{~J}_{m+1}\left(k_{2} a\right)-N m(2 m-2) \mathrm{J}_{m}\left(k_{2} a\right)}{a^{2}} \\
& M_{4,4}=\frac{N\left(\left(-2 m^{2}+2 m+k_{3}^{2} a^{2}\right) \mathrm{J}_{m}\left(k_{3} a\right)-2 k_{3} \mathrm{~J}_{m+1}\left(k_{3} a\right) a\right)}{a^{2}}
\end{aligned}
$$

the right-hand side vector comes from the contribution of the incident plane wave

$$
\begin{aligned}
& F_{1}=2 A_{i n c} k_{a}(-j)^{m} \mathrm{~J}_{m+1}\left(k_{a} a\right)-\frac{2 A_{i n c}}{a(-j)^{m} m \mathrm{~J}_{m}\left(k_{a} a\right)} \\
& F_{2}=-2 A_{i n c} \rho_{a} \omega^{2}(-j)^{m} \mathrm{~J}_{m}\left(k_{a} a\right) \\
& F_{3}=0 \\
& F_{4}=0
\end{aligned}
$$

These expressions can be found in [35] but with different coefficients.

\section{References}

[1] Y. Champoux, J. Allard, Dynamic tortuosity and bulk modulus in air-saturated porous media, J. Appl. Phys 70 (4) (1991) 1975-1979. doi:10.1063/1.349482.

[2] D. L. Johnson, J. Koplik, R. Dashen, Theory of dynamic permeability and tortuosity in fluid-saturated porous media, J. Fluid Mech 176 (1987) 379-402. doi:10.1017/ S0022112087000727.

[3] M. A. Biot, Theory of Propagation of Elastic Waves in a Fluid-Saturated Porous Solid. I. Low-Frequency Range, J. Acoust. Soc. Am. 28 (2) (1956) 168-178. doi:10.1121/1.1908239. 
[4] M. A. Biot, Mechanics of deformation and acoustic propagation in porous media, J. Appl. Phys (1962) 1482-1497.

[5] N. Atalla, R. Panneton, P. Debergue, A mixed displacement-pressure formulation for poroelastic materials, J. Acoust. Soc. Am. 104 (3) (1998) 1444-1452. doi:10.1121/1.424355.

[6] O. Dazel, B. Brouard, C. Depollier, S. Griffiths, An alternative Biot's displacement formulation for porous materials, J. Acoust. Soc. Am. 121 (6) (2007) 3509-3516.

[7] A. Deraemaeker, I. Babus, P. Bouillard, Dispersion and pollution of the FEM solution for the Helmholtz equation in one, two and three dimensions, Int. J. Numer. Meth. Engng (1999) 471-499.

[8] N. Dauchez, S. Sahraoui, N. Atalla, Convergence of poroelastic finite elements based on Biot displacement formulation, J. Acoust. Soc. Am. 109 (1) (2001) 33-40. doi:10.1121/1.1289924.

[9] N.-E. Hörlin, M. Nordström, P. Göransson, A 3-D hierarchical FE formulation of Biot's equations for elasto-acoustic modeling of porous media, J. Sound Vib. 245 (4) (2001) 633-652. doi:10.1006/jsvi.2000.3556

[10] N.-E. Hörlin, 3D hierarchical -FEM applied to elasto-acoustic modelling of layered porous media, J. Sound Vib. 285 (1-2) (2005) 341-363. doi:10.1016/j.jsv.2004.08.032.

[11] O. Cessenat, B. Despres, Application of an Ultra Weak Variational Formulation of Elliptic PDES to the Two-Dimensional Helmholtz Problem, SIAM J. Numer. Anal 35 (1) (1998) 255299.

[12] T. Strouboulis, I. Babuška, R. Hidajat, The generalized finite element method for Helmholtz equation: Theory, computation, and open problems, Comput. Methods Appl. Mech. Engrg. 195 (37-40) (2006) 4711-4731. doi:10.1016/j.cma.2005.09.019

[13] J.-D. Chazot, B. Nennig, E. Perrey-Debain, Performances of the Partition of Unity Finite Element Method for the analysis of two-dimensional interior sound fields with absorbing materials, J. Sound Vib. 332 (8) (2013) 1918-1929. doi:10.1016/j.jsv.2012.06.016.

[14] E. Deckers, N.-E. Hörlin, D. Vandepitte, W. Desmet, A Wave Based Method for the efficient solution of the 2D poroelastic Biot equations, Comput. Methods Appl. Mech. Engrg. 201-204 (2012) 245-262. doi:10.1016/j.cma.2011.09.015.

[15] G. Gabard, O. Dazel, A discontinuous Galerkin method with plane waves for sound-absorbing materials: plane-wave DGM for poro-elastic material, Int. J. Numer. Meth. Engng 104 (12) (2015) 1115-1138. doi:10.1002/nme.4961.

[16] J.-D. Chazot, E. Perrey-Debain, B. Nennig, The Partition of Unity Finite Element Method for the simulation of waves in air and poroelastic media, J. Acoust. Soc. Am. 135 (2) (2014) 724-733. doi:10.1121/1.4845315.

[17] W. G. Facco, E. J. Silva, R. Adriano, A. S. Moura, N. Z. Lima, Handling material discontinuities in a nonconforming generalized finite element method to solve wave propagation problems, Microw. Opt. Technol. Lett. 54 (12) (2012) 2709-2716. doi:10.1002/mop.27166.

[18] D. N. Arnold, An Interior Penalty Finite Element Method with Discontinuous Elements, SIAM J. Numer. Anal. 19 (4) (1982) 742-760. doi:10.1137/0719052. 
[19] P. Hansbo, J. Hermansson, Nitsche's method for coupling non-matching meshes in fluidstructure vibration problems, Comput. Mech 32 (1-2) (2003) 134-139. doi:10.1007/ s00466-003-0467-7.

[20] N. Moes, J. Dolbow, T. Belytschko, A finite element method for crack growth without remeshing, Int. J. Numer. Meth. Engng 46 (1999) 131-150.

[21] N. Sukumar, D. L. Chopp, N. Moës, T. Belytschko, Modeling holes and inclusions by level sets in the Extended Finite-Element Method, Comput. Methods Appl. Mech. Engrg. 190 (2001) $6183-6200$.

[22] N. Moës, M. Cloirec, P. Cartraud, J.-F. Remacle, A computational approach to handle complex microstructure geometries, Comput. Methods Appl. Mech. Engrg. 192 (28-30) (2003) 31633177. doi:10.1016/S0045-7825(03)00346-3.

[23] K. Dréau, N. Chevaugeon, N. Moës, Studied X-FEM enrichment to handle material interfaces with higher order finite element, Comput. Methods Appl. Mech. Engrg. 199 (2010) 1922-1936.

[24] G. Legrain, N. Chevaugeon, K. Dréau, High order X-FEM and levelsets for complex microstructures: Uncoupling geometry and approximation, Comput. Methods Appl. Mech. Engrg. 241244 (2012) 172-189. doi:10.1016/j.cma.2012.06.001.

[25] G. Legrain, A NURBS enhanced extended finite element approach for unfitted CAD analysis, Comput Mech 52 (4) (2013) 913-929. doi:10.1007/s00466-013-0854-7.

[26] A. Legay, The extended finite element method combined with a modal synthesis approach for vibro-acoustic problems, Int. J. Numer. Meth. Engng 101 (5) (2015) 329-350. doi:10.1002/ nme.4798,

[27] A. Gerstenberger, W. A. Wall, An eXtended Finite Element Method/Lagrange multiplier based approach for fluid-structure interaction, Comput. Methods Appl. Mech. Engrg. 197 (2008) 1699-1714.

[28] J.-F. Allard, N. Atalla, Propagation of Sound in Porous Media: Modelling Sound Absorbing Materials, 2nd Edition, Wiley, 2009.

[29] J. Melenk, I. Babuška, The partition of unity finite element method: Basic theory and applications, Comput. Methods Appl. Mech. Engrg. 139 (1-4) (1996) 289-314. doi:10.1016/ S0045-7825(96)01087-0

[30] T. Belytschko, C. Parimi, N. Moës, N. Sukumar, S. Usui, Structured extended finite element methods for solids defined by implicit surfaces: structured eXtended Finite Element Methods for solids, Int. J. Numer. Meth. Engng. 56 (4) (2003) 609-635. doi:10.1002/nme.686.

[31] O. Dazel, Numerical methods for the Biot theory in acoustics, HDR thesis, University of Maine-Le Mans (2011).

[32] O. Dazel, B. Brouard, J.-P. Groby, P. Göransson, A normal modes technique to reduce the order of poroelastic models: Application to 2D and coupled 3D models: comonent mode synthesis techniques for poroelastic materials, Int. J. Numer. Meth. Engng 96 (2) (2013) 110 128. doi:10.1002/nme.4551.

[33] A. Lieu, G. Gabard, H. Bériot, A comparison of high-order polynomial and wave-based methods for Helmholtz problems, J. Comput. Phys. 321 (2016) 105-125. doi:10.1016/j.jcp. 2016.05.045. 
[34] O. Axelsson, J. Karátson, F. Magoulès, Superlinear convergence using block preconditioners for the real system formulation of complex Helmholtz equations, J. Comput. Appl. Math. (2018) 424-431.

[35] B. Nennig, E. Perrey-Debain, J.-D. Chazot, The method of fundamental solutions for acoustic wave scattering by a single and a periodic array of poroelastic scatterers, Eng. Anal. Bound. Elem. 35 (8) (2011) 1019-1028. doi:10.1016/j.enganabound.2011.03.007. 